\title{
FINAL PROJECT REPORT
}

Project Title: DEVELOPMENT AND VALIDATION OF TEMPERATURE DEPENDENT THERMAL NEUTRON SCATTERING LAWS FOR APPLICATIONS AND SAFETY IMPLICATIONS IN GENERATION IV REACTOR DESIGNS

Covering Period: $\quad$ May $15^{\text {th }}, 2002$ through December $31^{\text {st }}, 2007$

Date of Report: June 20, 2008

Recipient: $\quad$ North Carolina Sate University

P.O. Box 7909

Department of Nuclear Engineering

Raleigh, NC 27695-7909

Award Number: DE-FG07-02SF22514 (NERI 01-140)

Subcontractors: N/A

Other Partners: $\quad$ Oak Ridge National Laboratory

Institute Balseiro, Argentina

Contact: $\quad$ Ayman I. Hawari

(919) 515-4598

ayman.hawari@ncsu.edu

Project Objective: The overall objectives of this project are to critically review the currently used thermal neutron scattering laws for various moderators as a function of temperature, select a well documented and representative set of experimental data sensitive to the neutron spectra to generate a data base of benchmarks, update models and models parameters by introducing new developments in thermalization theory and condensed matter physics into various computational approaches in establishing the scattering laws, benchmark the results against the experimental set. In the case of graphite, a validation experiment is performed by observing neutron slowing down as a function of temperatures equal to or greater than room temperature. In addition, an analysis is performed to understand the implications of the obtained results on the ability to accurately determine the operating and safety characteristics (e.g., the moderator temperature coefficient of reactivity, buildup and depletion of transuranium elements) of given reactor designs, which include Generation IV nuclear power systems, advanced research reactors and power reactors for space applications. 
Background: $\quad$ This project performs a thorough evaluation and update of the thermal neutron scattering kernels for several materials that are of interest in the nuclear technology field. This includes materials of primary interest to modern reactor concepts such as graphite, zirconium hydride, beryllium, beryllium oxide, light water and polyethylene, which are well known neutron moderators/reflectors. The work includes updating the computational models that are used in current thermal neutron calculations, and introducing modern Ab-Initio methods for computing the fundamental vibrational data that are needed to generate the scattering kernel for crystalline materials. A neutron slowing-down-time experiment will be conducted to benchmark the temperature dependent scattering kernel in graphite. Finally, an assessment will be made of the potential implications of the results on the operating characteristics of reactors.

Summary:

The project resulted in a general refinement of the treatment of thermal neutron scattering for the studied materials. For materials that are considered to be strong coherent scatterers of neutrons (e.g., graphite and beryllium), a more complete theoretical approach was developed and implemented for calculating inelastic thermal neutron scattering cross sections. In addition, a general ab initio approach for obtaining the basic information needed for thermal scattering calculations (e.g., the phonon frequency distributions) was developed. Furthermore, experimental measurements were performed to benchmark thermal neutron interactions in graphite. A transmission measurement was performed at the National Institute of Standards and Technology using $1 \mathrm{meV}$ neutrons. This measurement produced total cross section values (below the Bragg Cut-off) for samples of pyrolitic and reactor grade graphite. A slowing-down-time experiment was performed at the ORELA facility of ORNL to benchmark the behavior of neutrons as they slow-down and thermalize in a $70 \times 70 \times 70 \mathrm{~cm}$ graphite structure. This experiment revealed discrepancies of approximately $20 \%$ between measured and computationally predicted reaction rates (in the thermal energy range) for detectors placed outside the graphite structure. The discrepancies are attributed to the graphite thermal neutron scattering libraries that were implemented in the computations.

\section{Theoretical Background}

\subsection{Thermal Neutron Scattering}

Thermal neutrons have de Broglie wavelengths that are comparable to the inter-atomic spacing of the scattering material. In addition, the kinetic energy of slow neutrons is comparable to the energy levels that can be excited in a scattering event (e.g., the vibrational levels in graphite). To 
describe thermal neutron scattering in matter, the first Born approximation can be used to calculate the transition probability of the neutron-medium initial state to the neutron-medium final state. Since the neutron-nucleus interaction has a very short range, the interaction potential can be described by a delta function known as the Fermi pseudo-potential. Furthermore, the double differential scattering cross sections can be written as a sum of two terms representing the coherent and incoherent scattering cross sections. Based on these assumptions, the derivation of the expression for the double differential thermal neutron scattering cross section can be found in various standard references [1]. In compact form, the cross section is given by

$$
\frac{\mathrm{d}^{2} \sigma}{\mathrm{d} \Omega \mathrm{dE} E^{\prime}}=\frac{1}{4 \pi} \frac{\mathrm{k}^{\prime}}{\mathrm{k}}\left\{\sigma_{\mathrm{coh}} \mathrm{S}(\overrightarrow{\mathrm{Q}}, \omega)+\sigma_{\mathrm{inc}} \mathrm{S} \text { s }(\overrightarrow{\mathrm{Q}}, \omega)\right\}
$$

where $\mathrm{k}$ and $\mathrm{k}^{\prime}$ are the magnitude of the incident and scattered wave vectors of the neutron, $\sigma_{\text {coh }}$, and $\sigma_{\text {inc }}$ are the bound atom coherent and incoherent scattering cross section, $\mathrm{s}(\overrightarrow{\mathrm{Q}}, \omega)$ is known as the scattering law, $\overrightarrow{\mathrm{Q}}=\overrightarrow{\mathrm{k}}-\overrightarrow{\mathrm{k}}^{\prime}$ is the scattering vector, and $\hbar \omega$ is the energy lost by the neutron. $S(\vec{Q}, \omega)$ may be written as a sum of two parts: $S=S_{d}+S_{s}$, where $S_{s}$ is known as the self scattering law, it accounts for non-interference (incoherent) effects, and $S_{d}$ is the distinct scattering law accounting for interference (coherent) effects. If the atoms are assumed to be bound by harmonic forces, an expansion can be performed to allow the decomposition of the coherent and incoherent double differential scattering cross section into elastic and inelastic components. In crystalline materials, this expansion is known as a phonon expansion, as a consequence $S_{s}$ and $S_{d}$ can be written as

$$
\begin{aligned}
& \mathrm{S}_{\mathrm{s}}={ }^{0} \mathrm{~S}_{\mathrm{s}}+{ }^{1} \mathrm{~S}_{\mathrm{s}}+{ }^{2} \mathrm{~S}_{\mathrm{s}}+\cdots \\
& \mathrm{S}_{\mathrm{d}}={ }^{0} \mathrm{~S}_{\mathrm{d}}+{ }^{1} \mathrm{~S}_{\mathrm{d}}+{ }^{2} \mathrm{~S}_{\mathrm{d}}+\cdots
\end{aligned}
$$

Where the superscripts represent the number of phonons created or annihilated in the scattering process. To simplify the calculations, it is customary to use the incoherent approximation and neglect $S_{d}$. Therefore, Eq. (1) becomes

$$
\frac{\mathrm{d}^{2} \sigma}{\mathrm{d} \Omega \mathrm{dE}^{\prime}}=\frac{1}{4 \pi} \frac{\mathrm{k}^{\prime}}{\mathrm{k}}\left[\sigma_{\text {coh }}+\sigma_{\text {incoh }}\right] \sum_{\mathrm{P}=1}{ }^{\mathrm{P}} \mathrm{S}_{\mathrm{s}}(\overrightarrow{\mathrm{Q}}, \omega)
$$


Calculations of the scattering law for reactor moderators are normally performed under the incoherent approximations (i.e., Eq. (3)), using computer codes such as GASKET [2], and NJOY (LEAPR module) [3]. The basic input to Eq. (3) depends on the dynamics of the crystal, and is described by the phonon frequency distribution, $\rho(\beta)$. However, for materials such as graphite and beryllium, which are characterized by a strong coherent scattering behavior, it is observed that the thermal neutron scattering cross sections reported in libraries such as the latest ENDF/B-VII release [4] deviate from published measured data [5]. Consequently, as a first order correction for this discrepancy, the coherent 1-phonon term (i.e., ${ }^{1} S_{d}$ in Eq. (2)) is introduced. Therefore, noting that for materials such as beryllium and graphite $\sigma_{\text {inc }} \approx 0$, Eq. (3) can be rewritten as

$$
\frac{\mathrm{d}^{2} \sigma}{\mathrm{d} \Omega \mathrm{dE}^{\prime}}=\frac{1}{4 \pi} \frac{\mathrm{k}^{\prime}}{\mathrm{k}} \sigma_{\text {coh }}\left\{\left(\sum_{\mathrm{P}=2}{ }^{\mathrm{P}} \mathrm{S}_{\mathrm{s}}\right)_{\text {incoh }}+\left({ }^{1} \mathrm{~S}_{\mathrm{s}}+{ }^{1} \mathrm{~S}_{\mathrm{d}}\right)_{\text {exact }}\right\} .
$$

In this case, the $P=1$ term under the incoherent approximation (Eq. 3 ) is replaced by the exact 1 -phonon scattering law $\left({ }^{1} S_{s}+{ }^{1} S_{d}\right)$ exact . The exact term is given by

$$
\begin{aligned}
& { }^{1}\left(\frac{\mathrm{d}^{2} \sigma}{\mathrm{d} \Omega \mathrm{dE} E^{\prime}}\right)_{\operatorname{coh}}=\frac{(2 \pi)^{3}}{2 \mathrm{~V}_{\circ}} \frac{\mathrm{k}^{\prime}}{\mathrm{k}} \sum_{j, \overrightarrow{\mathrm{q}}, \vec{\tau}} \frac{1}{\omega_{j}(\overrightarrow{\mathrm{q}})}\left|\mathrm{F}_{\mathrm{j}}(\overrightarrow{\mathrm{Q}})\right|^{2} \times \\
& \left\{\begin{array}{l}
\left\langle\mathrm{n}_{\mathrm{j}}(\overrightarrow{\mathrm{q}})+1\right\rangle \delta\left(\omega-\omega_{\mathrm{j}}(\overrightarrow{\mathrm{q}})\right) \delta(\overrightarrow{\mathrm{Q}}-\overrightarrow{\mathrm{q}}-\vec{\tau})+ \\
\left\langle\mathrm{n}_{\mathrm{j}}(\overrightarrow{\mathrm{q}})\right\rangle \delta\left(\omega+\omega_{\mathrm{j}}(\overrightarrow{\mathrm{q}})\right) \delta(\overrightarrow{\mathrm{Q}}+\overrightarrow{\mathrm{q}}-\vec{\tau})
\end{array}\right\}
\end{aligned} .
$$

$\mathrm{V}$ 。 is the volume of the unit cell in the crystal, and $F_{j}(\vec{Q})$ is given by

$$
\mathrm{F}_{\mathrm{j}}(\overrightarrow{\mathrm{Q}})=\sum_{\mathrm{d}} \frac{\overline{\mathrm{b}}_{\mathrm{d}}}{\sqrt{\mathrm{M}_{\mathrm{d}}}}\left(\overrightarrow{\mathrm{Q}} \cdot \overrightarrow{\mathrm{e}}_{\mathrm{dj}}(\overrightarrow{\mathrm{q}})\right) \mathrm{e}^{-\mathrm{W}_{\mathrm{d}}} \mathrm{e}^{\mathrm{i} \overrightarrow{\mathrm{Q}} \cdot \overrightarrow{\mathrm{d}}}
$$

where $\vec{d}$ is the equilibrium position of atom $d$ in the unit cell, $j$ is the polarization index $(=1,2,3), M_{d}$ is the mass of atom $d, \vec{q}$ is the wave vector, $\omega_{j}(\vec{q})$ is the angular frequency, $n_{j}(\vec{q})$ is the quantum number of the normal mode $(j, \vec{q}), \vec{e}_{j d}(\vec{q})$ is the polarization vector of normal mode $(j, \vec{q})$ of atom $d, \vec{\tau}$ is the reciprocal lattice vector, and $e^{-2 W_{d}}$ is the Debye-Waller factor. Equation (5) has two parts, the first part represents phonon creation (+) while the second part represents phonon annihilation (-). To 
make the above equation computationally tractable a numerical approach similar to that presented in [6] was used. In this case, the summation over $\overrightarrow{\mathrm{q}}$ and $\vec{\tau}$ was replaced by an integral over $\overrightarrow{\mathrm{Q}}$. Also, it is more convenient to write the momentum and energy transfer in terms of the dimensionless variables

$$
\alpha=\frac{E^{\prime}+E-2 \sqrt{E E^{\prime} \mu}}{\mathrm{Ak}_{\mathrm{B}} \mathrm{T}} \quad \text { and } \quad \beta=\frac{\hbar \omega}{\mathrm{k}_{\mathrm{B}} \mathrm{T}}
$$

where $E$ and $E^{\prime}$ are the incident and scattered neutron energies, $\mu$ is the cosine of the scattering angle, $A$ is the ratio of the mass of the scattering atom to the neutron mass, $\mathrm{k}_{\mathrm{B}}$ is Boltzmann's constant, and $\mathrm{T}$ is the temperature in Kelvin. As a result, the scattering law can be written in terms of $\alpha$ and $\beta$ as

$$
S(\alpha, \beta)=\mathrm{k}_{\mathrm{B}} \mathrm{Te} \mathrm{e}^{\beta / 2} \mathrm{~S}(\overrightarrow{\mathrm{Q}}, \omega) .
$$

Using Eq. (8) and replacing the angular frequency $\omega_{j}(\overrightarrow{\mathrm{q}})$ by $\beta$ and $\langle\mathrm{n}\rangle$ by $1 /\left(e^{\beta}-1\right)$, and assuming that for a small interval, $\Delta \beta$, the delta function can be approximated by $1 / \Delta \beta$, the annihilation term (-) in Eq. 5 can be written as

$$
{ }^{1} S_{-}(\alpha, \beta)=\frac{\hbar^{2}}{2 \mathrm{nk}_{\mathrm{B}} \mathrm{T}} \frac{1}{2 \beta \sinh (\beta / 2)} \sum_{\mathrm{j}, \mathrm{Q}} \frac{\left|\mathrm{F}_{\mathrm{j}}(\overrightarrow{\mathrm{Q}})\right|^{2}}{\mathrm{l}(\alpha, \Delta \alpha)} \frac{1}{\Delta \beta},
$$

where $1(\alpha, \Delta \alpha)$ is the number of mesh points in the interval $\Delta \alpha$. Utilizing the principle of detailed balance, the creation term (+) can be then be written as

$$
{ }^{1} S_{+}(\alpha,-\beta)=e^{\beta}{ }^{1} S_{-}(\alpha, \beta) .
$$

From the above, it can be seen that evaluating the scattering law, starts by evaluating the sum in equation (9), where the polarization vectors and dispersion relations are required as input to the structure factor. In principle, it is necessary to sweep all reciprocal space to perform the summation over $\overrightarrow{\mathrm{Q}}$. However, as a consequence of crystal symmetry, in any region in reciprocal space, the polarization vectors $\overrightarrow{\mathrm{e}}_{\mathrm{jd}}(\overrightarrow{\mathrm{q}})$ and the dispersion relations $\omega_{j}(\vec{q})$ are just a symmetric transformation of $\vec{e}_{j d}(\vec{q})$ and $\omega_{\mathrm{j}}(\overrightarrow{\mathrm{q}})$ in the first Brillouin zone. Therefore, the summation over $\vec{Q}$ can be evaluated in the first Brillouin zone. Once the coherent one- 
phonon scattering law is calculated, the thermal neutron scattering cross sections can be generated by utilizing Eq. (4).

\subsection{Ab Initio Lattice Dynamics}

To calculate the scattering cross section, the phonon frequency distribution $\rho(\beta)$ is the basic input for the formulation implemented in GASKET and LEAPR, based on Eq. (3), while the basic input for the coherent one-phonon scattering cross section, Eq. (5), are the dispersion relations $\omega_{j}(\vec{q})$ and the polarization vectors $\vec{e}_{d s}(\vec{q})$. In general, the first step in establishing the dispersion relations, and the phonon frequency distribution for crystalline materials, is to set up a dynamical matrix based on the crystal structure. In fact, this requires writing the equations of motion of atoms in the crystal. Usually, these equations are written utilizing the harmonic approximation. Using a plane wave solution to these equations one can get a finite set of linear differential equations

$$
\omega_{j}^{2}(\vec{q}) \vec{e}_{\theta d j}(\vec{q})=\sum_{d^{\prime}, \eta} D_{\theta \eta}\left(\vec{q} ; d, d^{\prime}\right) \vec{e}_{\eta d^{\prime} j}(\vec{q}),
$$

where $\theta$ and $\eta$ are $x, y$, or $z$ in Cartesian coordinates. $D_{\theta \eta}\left(\vec{q} ; d, d^{\prime}\right)$ are the coefficients of the dynamical matrix $\mathrm{D}(\overrightarrow{\mathrm{q}})$ and are given by

$$
\mathrm{D}_{\theta \eta}\left(\overrightarrow{\mathrm{q}} ; \mathrm{d}, \mathrm{d}^{\prime}\right)=\frac{1}{\sqrt{\mathrm{M}_{\mathrm{d}} \mathrm{M}_{\mathrm{d}^{\prime}}}} \sum_{\mathrm{l}^{\prime}} \Phi_{\theta \eta}\left(\mathrm{l}, \mathrm{d} ; \mathrm{l}^{\prime}, \mathrm{d}^{\prime}\right) \mathrm{e}^{-\mathrm{i} \mathrm{q} \cdot\left[\overrightarrow{\mathrm{R}}(\mathrm{l}, \mathrm{d})-\overrightarrow{\mathrm{R}}\left(\mathrm{l}^{\prime}, \mathrm{d}^{\prime}\right)\right]}
$$

$\Phi_{\theta \eta}\left(\mathrm{l}, \mathrm{d} ; \mathrm{l}^{\prime}, \mathrm{d}^{\prime}\right)$ is the atomic force constant, and $\overrightarrow{\mathrm{l}}$ represents the position vector of the $\mathrm{I}^{\text {th }}$ unit cell. Equation (11) has a nontrivial solution only when $\left|\mathrm{D}_{\theta \mu}\left(\overrightarrow{\mathrm{q}} ; \mathrm{d}, \mathrm{d}^{\prime}\right)-\omega_{\mathrm{j}}^{2}(\overrightarrow{\mathrm{q}}) \delta_{\theta \eta} \delta_{\mathrm{dd^{ \prime }}}\right|=0$. For each value of the wave vector $\overrightarrow{\mathrm{q}}$ there are $3 n$ solutions (branches) denoted by $\omega_{j}^{2}(\vec{q})$, where $n$ is the number of atoms per unit cell. Furthermore, for each value of $\omega_{j}^{2}(\vec{q})$, there is a polarization vector $\vec{e}_{d j}(\vec{q})$ whose components are the solutions to the set of Equations (12) [7].

Traditionally, phonons have been calculated by proposing an analytic model for the interaction between atoms, and evaluating the force constants between atoms by fitting to experimental data. This approach suffers from two major deficiencies. The first is that it is not predictive, producing atomic force constants and dispersion relations that are 
inferred from experimental data. The second is that the results are not unique and can possibly be reproduced by alternative dynamical models. An alternative way to calculate the atomic force constants is by utilizing the ab initio (first principle) approach, in which the analytic model is replaced by full quantum mechanic electronic structure calculation. In this case, density functional theory (DFT) $[8,9]$ is applied to replace the manyelectron problem by an exactly equivalent set of self-consistent Shrödinger like one-electron equations known as the Khon-Sham equations

$$
\hat{\mathrm{H}}_{\mathrm{KS}} \psi_{\mathrm{i}}=\varepsilon_{\mathrm{i}} \psi_{\mathrm{i}},
$$

where $\psi_{\mathrm{i}}$ is the wavefunction of electronic state $\mathrm{i}, \varepsilon_{\mathrm{i}}$ is the Kohn-Sham eigenvalue, and $\hat{\mathrm{H}}_{\mathrm{KS}}$ is the Kohn-Sham Hamiltonian that corresponds to an energy functional given by

$$
E[n(\vec{r})]=T_{\circ}[n(\vec{r})]+E_{\text {ion }}[n(\vec{r})]+E_{H}[n(\vec{r})]+E_{X C}[n(\vec{r})] .
$$

$n(\vec{r})$ is the electronic density of the system, $T_{\circ}[n(\vec{r})]$ is the kinetic energy functional of the non-interacting electron gas, $\mathrm{E}_{\text {ion }}[\mathrm{n}(\overrightarrow{\mathrm{r}})]$ is the electron-ion interaction energy functional, $E_{H}[n(\vec{r})]$ is the Hartree energy functional, and $E_{X C}[n(\vec{r})]$ is the exchange-correlation energy functional. In addition, Hellmann-Feynman theorem [10] simplifies the calculations of the physical forces on the ions. It states that for any perturbation $\lambda$, the first derivative of the ground state energy of the Hamiltonian $\mathrm{H}_{\lambda}$, i.e. $\mathrm{E}_{\lambda}$, with respect to $\lambda$ is given by

$$
\frac{\mathrm{dE}_{\lambda}}{\mathrm{d} \lambda}=\left\langle\psi_{\lambda}\left|\frac{\partial \mathrm{H}_{\lambda}}{\partial \lambda}\right| \psi_{\lambda}\right\rangle
$$

If $\lambda_{d}$ represents the displacement of the $d^{\text {th }}$ ion, then Eq. (15) represents the force acting on the $d^{\text {th }}$ nucleus. In the frame of DFT, the electronic wave functions must be eigenstates of the Kohn-Sham Hamiltonian so that Hellmann-Feynman theorem is applicable. The ab initio forceconstant direct approach (applied here) is based on the solution of the Kohn-Sham equation, where the phonon frequencies are calculated from Hellmann-Feynman forces generated by a small atomic displacement, once at a time. 


\section{Thermal Neutron Scattering Cross Section Calculations}

\subsection{Beryllium (Be)}

Beryllium belongs to the space group P6_3/mmc. It has a hexagonal closed packed structure with two atoms per unit cell located at $(1 / 3,2 / 3,1 / 4)$, and $(2 / 3,1 / 3,3 / 4)$ the lattice constants values are $a=b=$ $2.29 \AA$, and $c=3.58 \AA$. The calculations of the dispersion relations were performed using the Vienna Ab Inition Simulation Package (VASP) and the PHONON code [11,12]. The generalized gradient approximation (GGA), which accounts for variations in the electron density, was utilized. The projector augmented wave (PAW) pseudopotential was implemented assuming a $4 \times 4 \times 3$ supercell (96 atoms). The integration over the Brillouin zone was confined to a $4 \times 4 \times 4$ k-mesh points generated by the Monkhrust-Pack scheme. The Hellmann-Feynman forces were computed from 3 independent displacements along $x, y$ and $z$, each displacement generating 288 force components, for a total of 864 components. Figure 1 shows the beryllium unit cell and the ab initio based dispersion relations for beryllium metal $(\mathrm{Be})$ compared to data generated using neutron scattering experiments [13]. Six vibrational modes appear in the figure as expected for the beryllium unit cell, which has 2 atoms. The lowest three branches, starting from $\Gamma$, are known as the acoustic modes, while the highest three branches are the optical modes. To compare to measurement, the dispersion relations are calculated along the high symmetry directions $(\Gamma, \mathrm{K}, \mathrm{M}, \mathrm{H}$, and $\mathrm{L})$ in the hexagonal Brillouin zone. As it can be seen, the agreement between measurement and calculation is excellent.
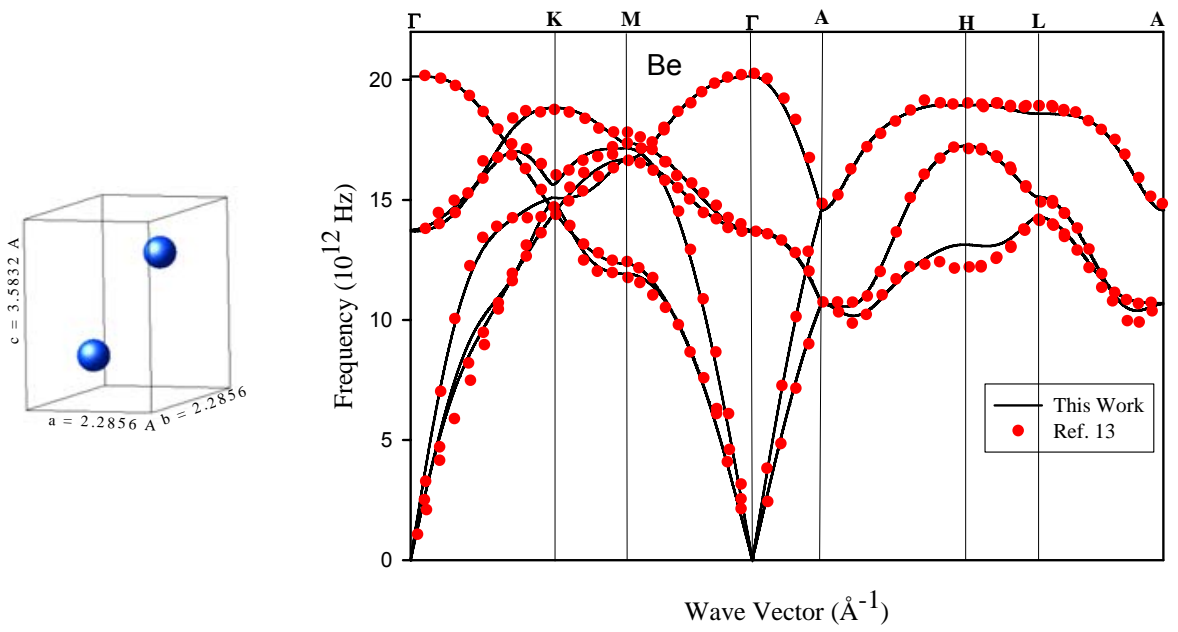

Fig. 1. The beryllium unit cell (left) and the dispersion relations (right). 
The calculation of the phonon frequency distribution for Beryllium can be performed once the dispersion relations are known. Figure 2 shows this distribution in comparison to the one utilized previously [14]. Applying the procedures outlined in section 1 the thermal neutron scattering cross section for beryllium can be calculated. Figure 3 shows the calculated cross section (using the methodology described in section 1.1 and Eq. 4) compared to ENDF/B-VII based data (section 1.1, Eq. 3) and experimental values [15]. Clearly, the approach developed in this work, which is based on using the full dispersion relations combined with the correction due 1-phonon coherent scattering, achieves the best agreement between calculations and experimental values.

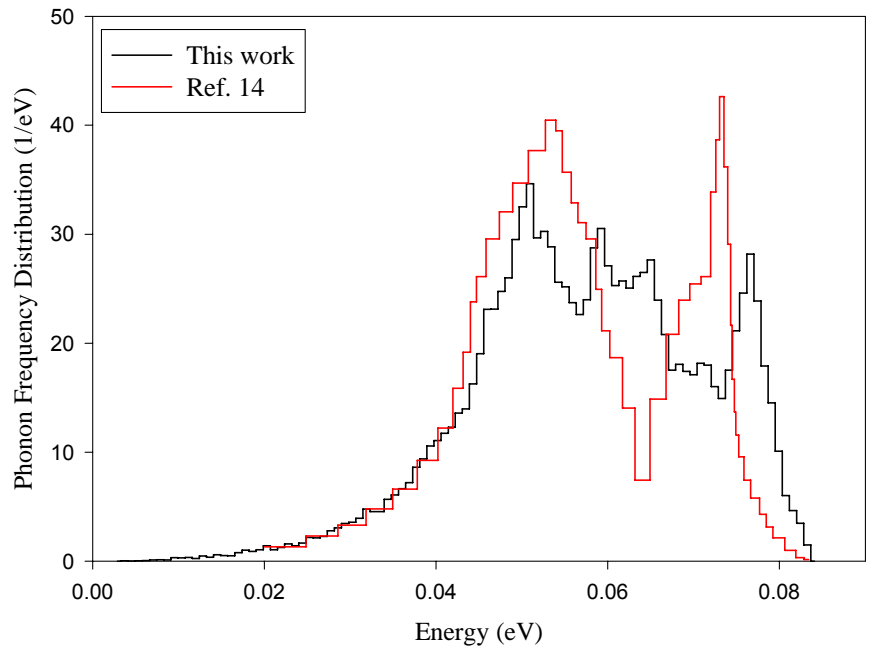

Fig. 2. The phonon frequency distribution for beryllium.

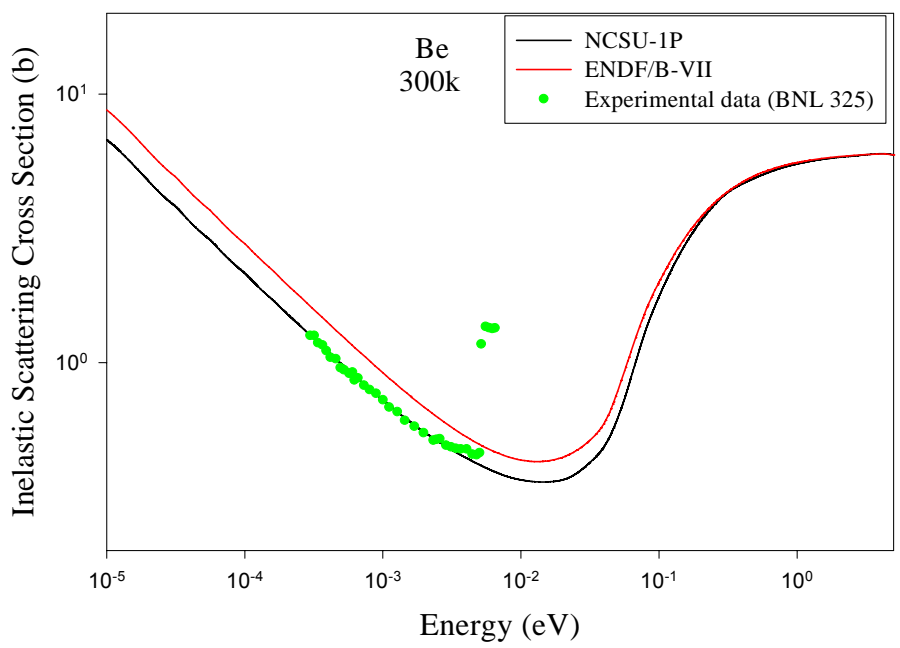

Fig 3. Total Cross Section for Beryllium at 300K. 


\subsection{Graphite}

The approach described above was implemented to calculate the thermal neutron scattering cross sections for graphite. As shown in Fig. 4, Graphite has a hexagonal structure that belongs to the space group P6_3/mmc, with four atoms per unit cell. Because of it is long c-axis, graphite has a unique structure, which results in anisotropic behavior in its properties (thermal, mechanical, etc.). The ab initio calculations of graphite were carried out using the local density approximation (LDA) of DFT, with the projector augmented wave (PAW) pseudopotential implemented by the Vienna Ab Initio Simulation Package (VASP) [11] and using an energy cutoff of $500 \mathrm{eV}$. The PHONON code [12] was used to calculate the dispersion relations and phonon frequency distributions shown in Fig. 5. The VASP/PHONON model, shown in Fig. 4, used a $6 \times 6 \times 1$ supercell composed of 144 atoms. The integration over the Brillouin zone was confined to a $3 \times 3 \times 4$ k-mesh generated by the Monkhrust-Pack scheme.
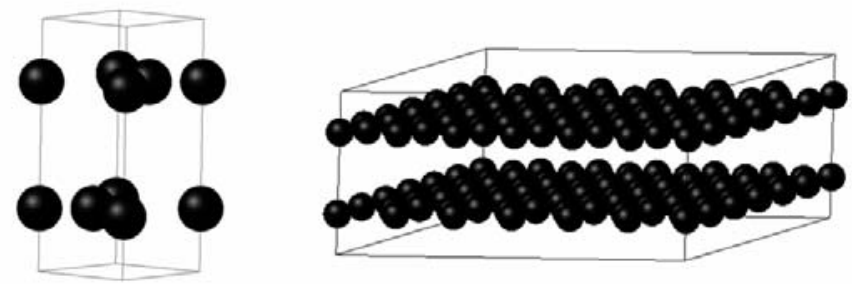

Fig. 4. The graphite unit cell (left) and supercell (right) used in the calculations.

Figure 5 shows the dispersion relations along the highest symmetry points of the Brillioun zone $(A, \Gamma, M, K$, and $\Gamma$ ). Since graphite has 4 atoms per unit cell, twelve vibrational modes appear in the figure as expected. For comparison, the figure also shows experimental data that were obtained through various techniques [16-21]. As it can be seen, the agreement is remarkable and clearly illustrates the power and utility of the $a b$ initio approach. The figure also shows the ab initio phonon frequency distribution for graphite compared to the one used in the current (ENDF/B-VII) compilations of thermal neutron scattering cross sections [22]. The agreement is reasonable, however, in the low energy region that is of interest in thermal neutron scattering calculations, the ab intio model shows higher frequency values. 

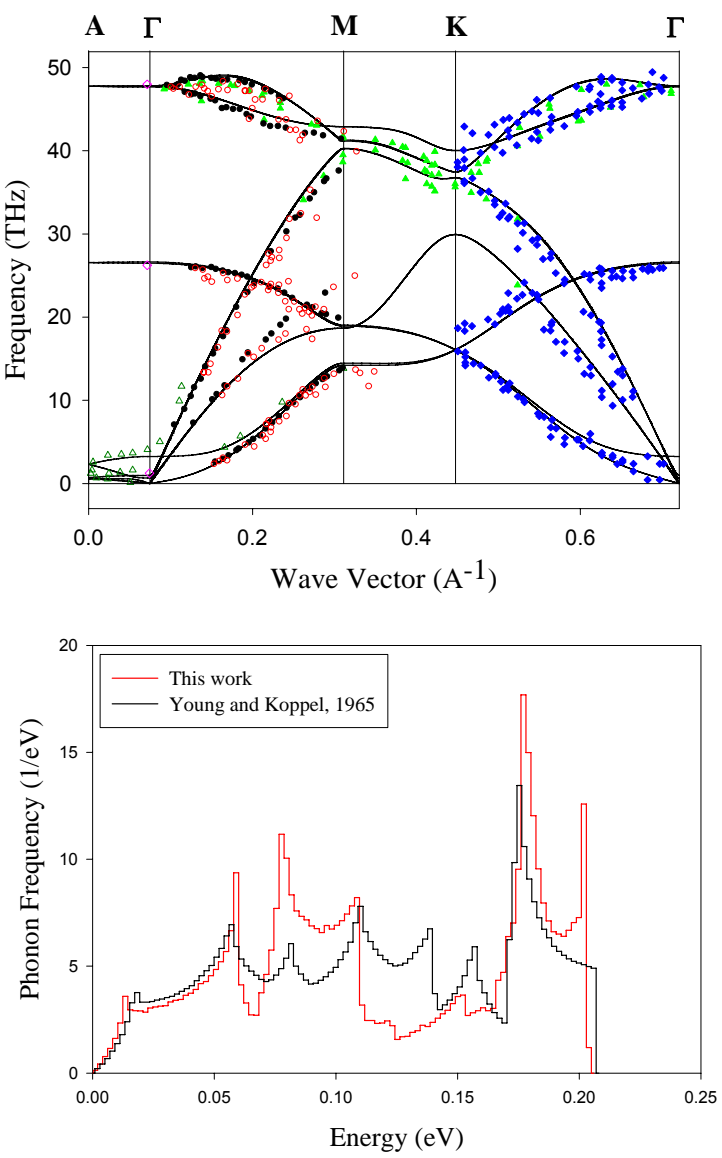

Fig. 5. Graphite dispersion relations (top) compared to experimental data. The solid lines are generated using $a b$ initio simulations and the lattice model of Fig. 4. The bottom plot shows the phonon frequency distribution of graphite.

Figures 6 shows the inelastic thermal neutron scattering cross sections of graphite at different temperatures generated by directly using the dispersion relations of Fig. 4 and Eq. (4). These cross sections are compared to the data generated using the phonon frequency spectra of Fig. 5 and Eq. 3. In addition, both calculated data sets are compared to experimental data belonging to pyrolytic graphite (density $\approx 2.2 \mathrm{~g} / \mathrm{cm}^{3}$ ) [5], and reactor grade graphite (density $\approx 1.7 \mathrm{~g} / \mathrm{cm}^{3}$ ). A third set of experimental data that is shown in the figure belongs to the BNL-325 database [15]. Recent measurements [23] using reactor grade graphite samples seem to indicate that the BNL-325 data points may have been generated using this type of material.

As it can be seen, good agreement exists between the results of Eq. 4 and the pyrolytic graphite data, while Eq. 3 seems to underestimate the 
experimental data. However, for the data associated with reactor grade type graphite, it seems that both equations underestimate the data. This can be clearly seen in Fig. 6 where the data was generated at a temperature of $300 \mathrm{~K}$. This deviation is attributed to the fact that reactor grade graphite is considered to be a composite material that is composed of graphite crystallites embedded in an amorphous-like carbon matrix. In addition, reactor grade graphite is a highly porous material (nearly $30 \%$ porosity fraction). All these effects will generate different lattice dynamics properties than what was originally assumed in the generation of the graphite thermal scattering libraries (e.g., in the ENDF/B-VII compilation). Nevertheless, it should be noted that at all temperatures the significant difference between the various libraries is in the 0.01 to $0.1 \mathrm{eV}$ energy range. This difference varies from $50 \%$ to $25 \%$ as the temperature increases from $300 \mathrm{~K}$ to $1020 \mathrm{~K}$ and results mainly from the differences in the phonon spectra (Fig. 5).

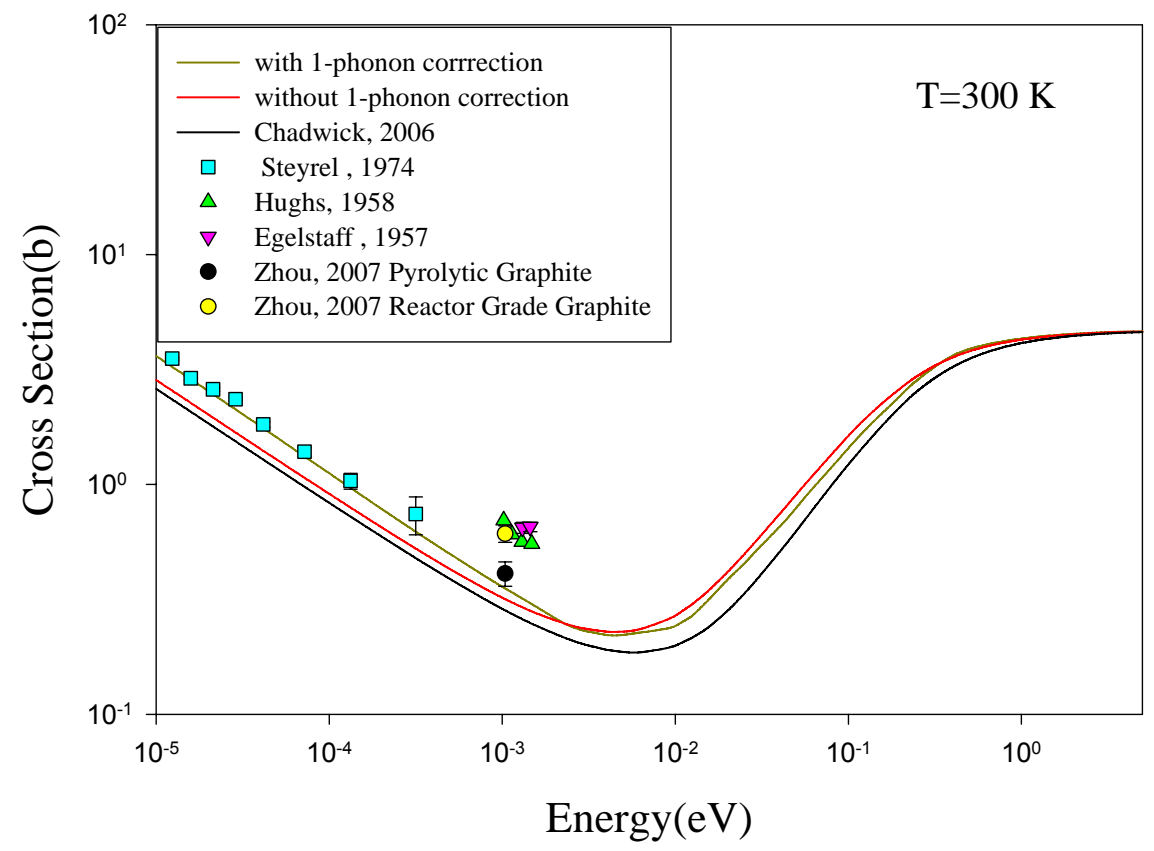

Fig. 6. The thermal neutron inelsatic scattering cross section of graphite as a function of temeprature. 

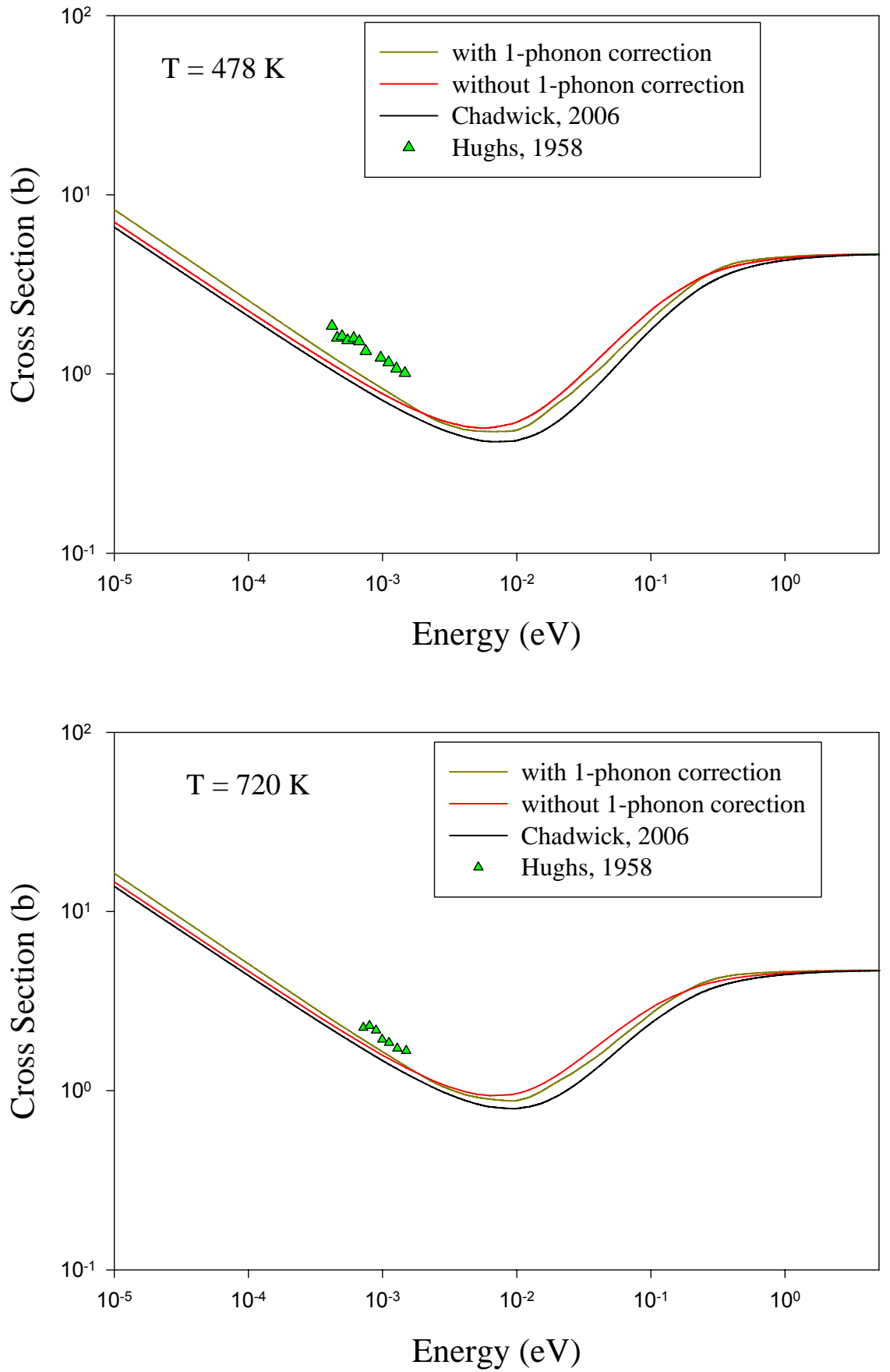

Fig. 6. continued. 


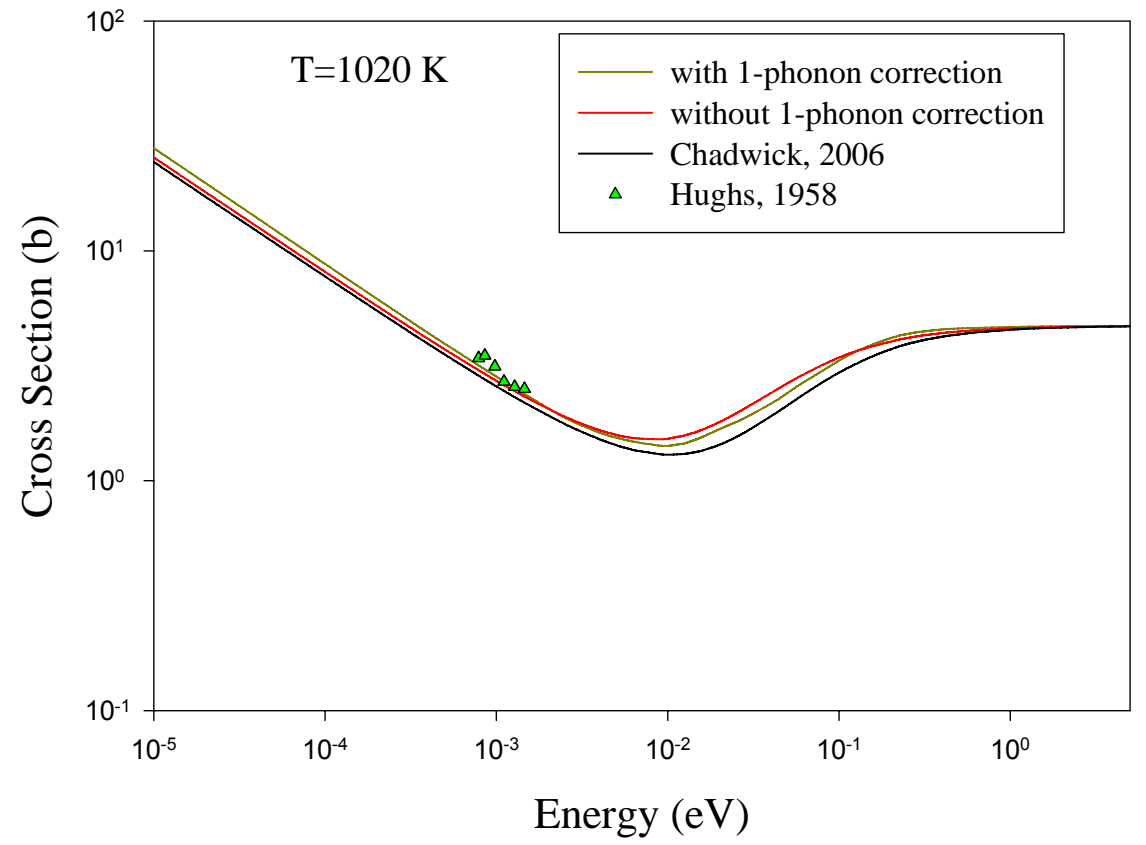

Fig. 6. continued.

\subsection{Zirconium Hydride $\left(\mathrm{ZrH}_{x}\right)$}

During this project, analysis was performed on zirconium hydride $\left(\mathrm{ZrH}_{\mathrm{x}}\right)$ as a function of the hydrogen content (i.e., $\mathrm{x}$ ). In this case, $\mathrm{ZrH}_{1.75}$ and $\mathrm{ZrH}_{2}$ were studied with both having the epsilon $(\varepsilon)$ phase and a body centered tetragonal (BCT) structure, which belongs to the space group $14 / \mathrm{mmm}$. $\mathrm{ZrH}_{2}$ has six atoms per unit cell (two $\mathrm{Zr}$ and four $\mathrm{H}$ ). In order to model $\mathrm{ZrH}_{1.75}$, a $2 \times 2 \times 1 \mathrm{ZrH}_{2}$ supercell was built that contains 24 atoms (eight $\mathrm{Zr}$ and sixteen $\mathrm{H}$ ). Two hydrogen atoms were removed randomly, that is the ratio of Hydrogen atoms to $Z r$ atoms was set to $22: 8=1.75: 1$. Figure 7 shows the $\mathrm{ZrH}_{2} 2 \times 2 \times 1$ supercell and $\mathrm{ZrH}_{1.75}$ supercell.

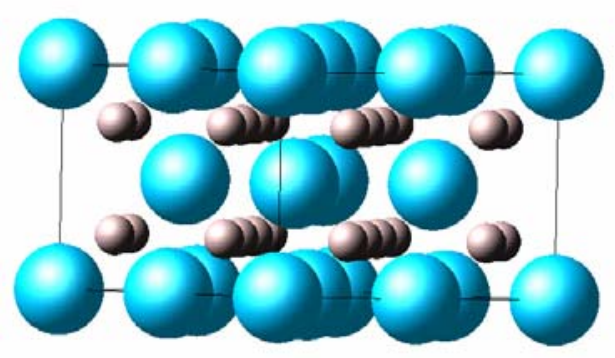

$\mathrm{ZrH}_{2}$ (24 atoms)

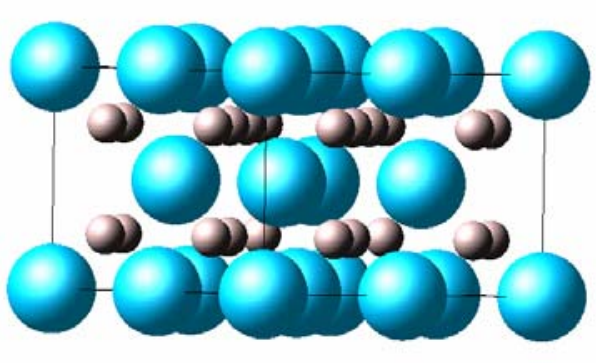

$\mathrm{ZrH}_{1.75}$ (22 atoms)

Fig 7. $\mathrm{ZrH}_{2}$ and $\mathrm{ZrH}_{1.75}$ cells. Notice the missing atoms in the cell of $\mathrm{ZrH}_{1.75}$. 
Figure 8 shows the phonon frequency distributions for the two structures. While the acoustic part in $\mathrm{ZrH}_{1.75}$ has almost the same shape as that in $\mathrm{ZrH}_{2}$ (except it is shifted towards lower energies), the optical part of $\mathrm{ZrH}_{1.75}$ is broadened and loses its Gaussian shape due to symmetry breaking by the hydrogen atoms.

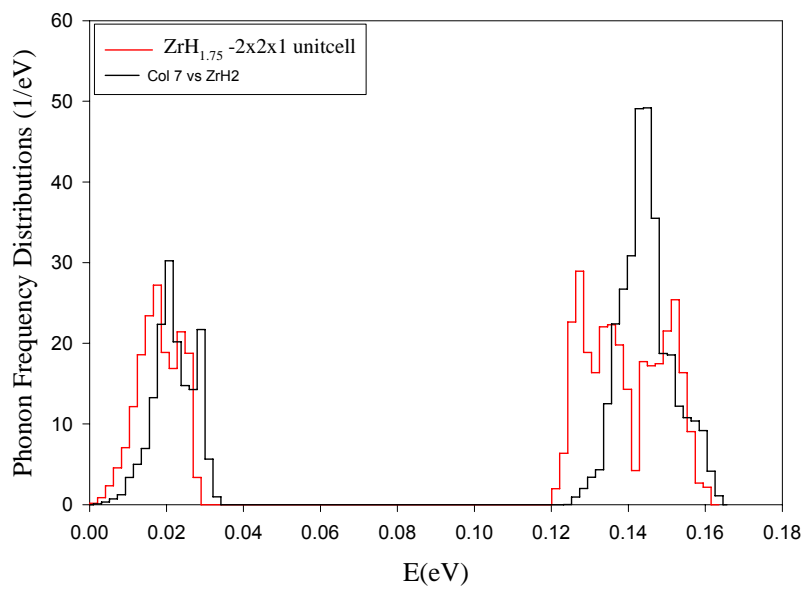

Fig 8. $\mathrm{ZrH}_{1.75}$ and $\mathrm{ZrH}_{2}$ phonon frequency distributions.

Figure 9 shows the partial phonon frequency distributions of $\mathrm{Zr}$ and $\mathrm{H}$ in $\mathrm{ZrH}_{\mathrm{x}}\left(\mathrm{x}=1.75\right.$ or 2.0). As it can be seen, the acoustic part of $\mathrm{Zr}$ in $\mathrm{ZrH}_{1.75}$ has a higher phonon frequency distribution at low energy, so we expect the inelastic scattering cross sections of $\mathrm{ZrH}_{1.75}$ to be higher than those of $\mathrm{ZrH}_{2}$ for the same temperature. This demonstrates the advantage of the ab initio apparoch; it can calculate the optical modes for heavy atoms, and the acoustical modes of the light atoms. Notice that in Fig. 9, the $\mathrm{Zr}$ optical modes have been multiplied by 100 for clarity.

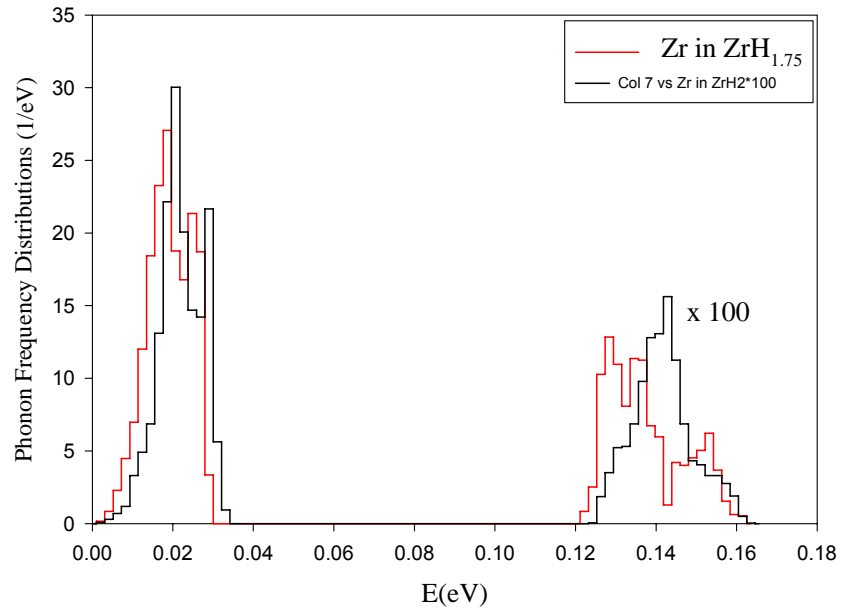

Fig 9. $\mathrm{Zr}$ in $\mathrm{ZrH}_{1.75}$ and $\mathrm{ZrH}_{2}$ phonon frequency distributions. 
Figure 10 shows the $\mathrm{H}$ phonon frequency distribution in $\mathrm{ZrH}_{\mathrm{x}}$. The $\mathrm{H}$ acoustical modes have been also multiplied by 100 for clarity.

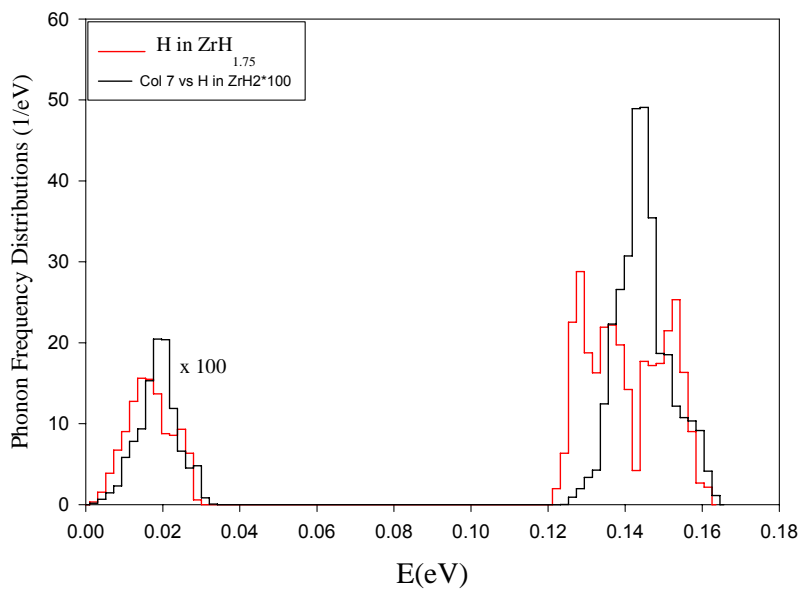

Fig 10. $\mathrm{H}$ in $\mathrm{ZrH}_{1.75}$ and $\mathrm{ZrH}_{2}$ phonon frequency distributions.

Using the above data, the thermal neutron inelastic cross section were calculated using the LEAPR/NJOY code system and the incoherent approximation (i.e., Eq. 3 of section 1.1). Figure 11 shows the inelastic scattering cross section of $\mathrm{Zr}$ in $\mathrm{ZrH}_{\mathrm{x}}$ at two different temperatures, the difference is clear especially in the energy range 0.001-0.1 eV, and depending on the temperature. Figure 12 shows the $\mathrm{H}$ inelastic scattering cross sections in $\mathrm{ZrH}_{\mathrm{x}}$ at $300 \mathrm{~K}$ and $800 \mathrm{~K}$.

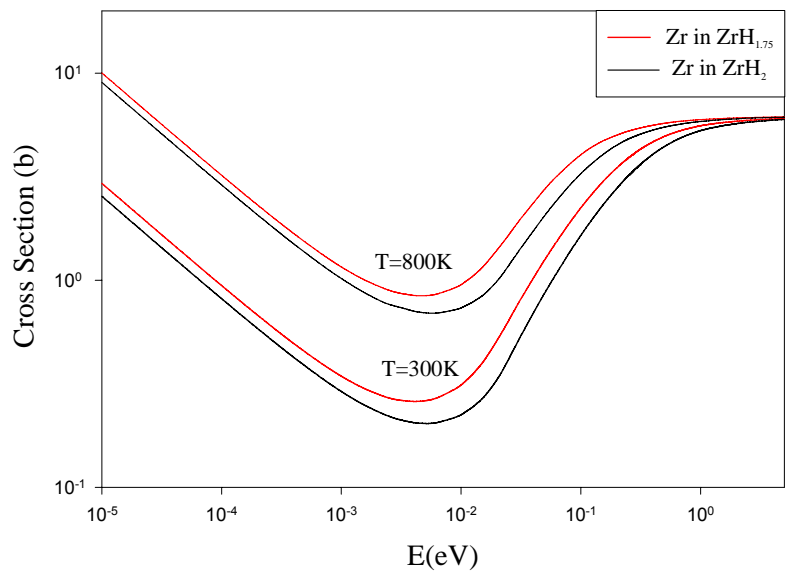

Fig 11. Inelastic scattering cross sections of $\mathrm{Zr}$ in $\mathrm{ZrH}_{1.75}$ and $\mathrm{ZrH}_{2}$. 


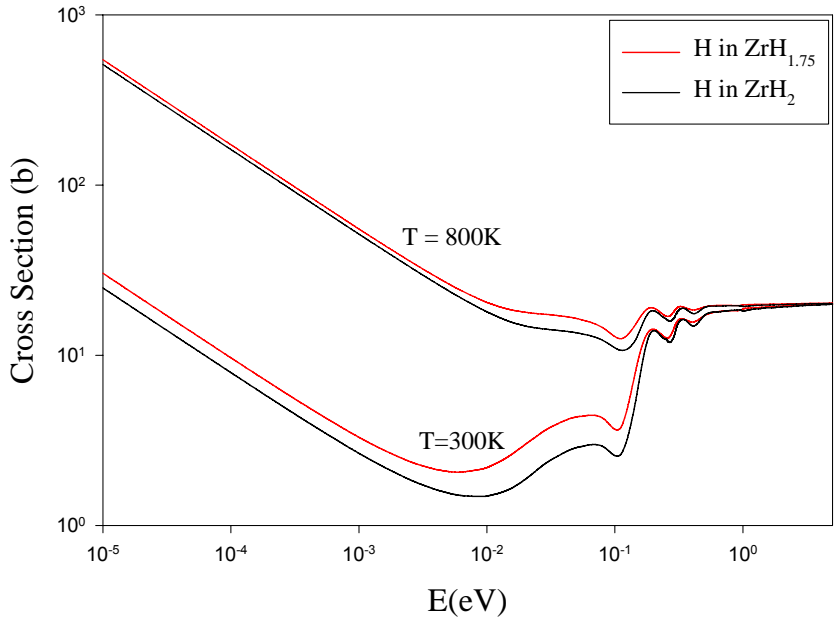

Fig 12. Inelastic scattering cross section of $\mathrm{H}$ in $\mathrm{ZrH}_{1.75}$ and $\mathrm{ZrH}_{2}$.

\subsection{Light Water $\left(\mathrm{H}_{2} \mathrm{O}\right)$}

The approach to calculating the thermal neutron inelastic scattering cross section is based on a treatment similar to that used in the NJOY/LEAPR reports [3]. In this case, a generalized frequency spectrum is introduced and used in the LEAPR module of NJOY. However, in this work the generalized spectrum was replaced by the low energy (below $0.2 \mathrm{eV}$ ) treatment of Edura and Morishima [24]. In addition, the proposed frequency spectrum for oxygen was introduced and used in generating the scattering cross sections. Figures 13 and 14 below show the frequency spectra for hydrogen and oxygen.

Thermal neutron scattering cross sections were generated using both the original frequency distribution and the newly constructed distribution at 296 K. Figure 15 compares the results of both calculations to the experimental values as shown in BNL-325. It can be seen that above $0.01 \mathrm{eV}$ both models compare favorably to the BNL data. Below 0.001 $\mathrm{eV}$ the Edura-Morishima spectrum shows slightly improved agreement.

\subsection{Beryllium Oxide (BeO)}

$\mathrm{BeO}$ has a wurtzite structure. It can be described as two separate, identical, and interpenetrating arrays of metal $(\mathrm{Be})$ and nonmetal $(\mathrm{O})$ atoms. Each array is hexagonal close-packed with the metal atom array shifted along the c-axis with respect to the nonmetal array. The crystal symmetry of the $\mathrm{BeO}$ structure is $C_{6 v}^{4}-P 6_{3} m c$. There are four atoms 
(two molecules) per unit cell. The Be atoms located at $(1 / 3,2 / 3,0)$ and $(2 / 3,1 / 3,1 / 2)$ and the $O$ atoms located at $(1 / 3,2 / 3, \delta)$ and $(2 / 3,1 / 3$, $0.5+\delta$ ). Figure 17 shows the unit cell used in the calculations.

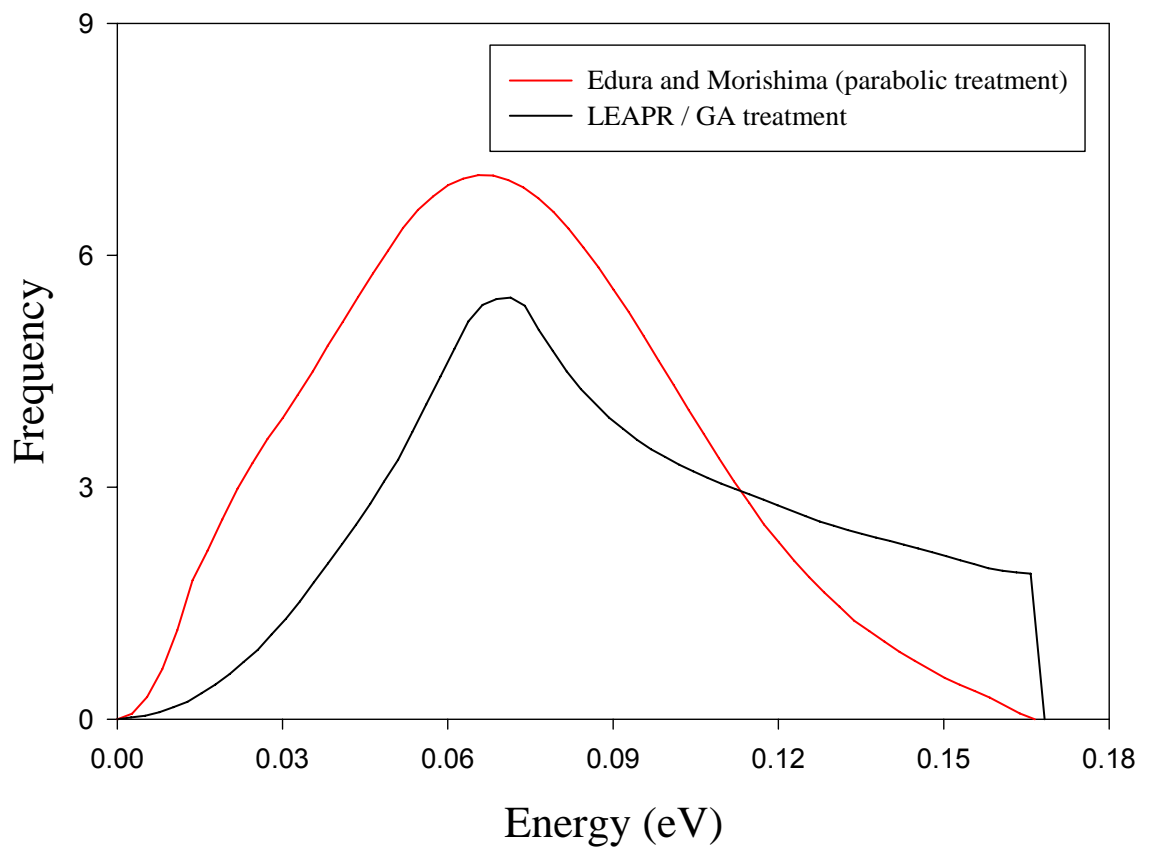

Fig. 13. Comparison of the frequency spectrum of $\mathrm{H}$ in $\mathrm{H}_{2} \mathrm{O}$.

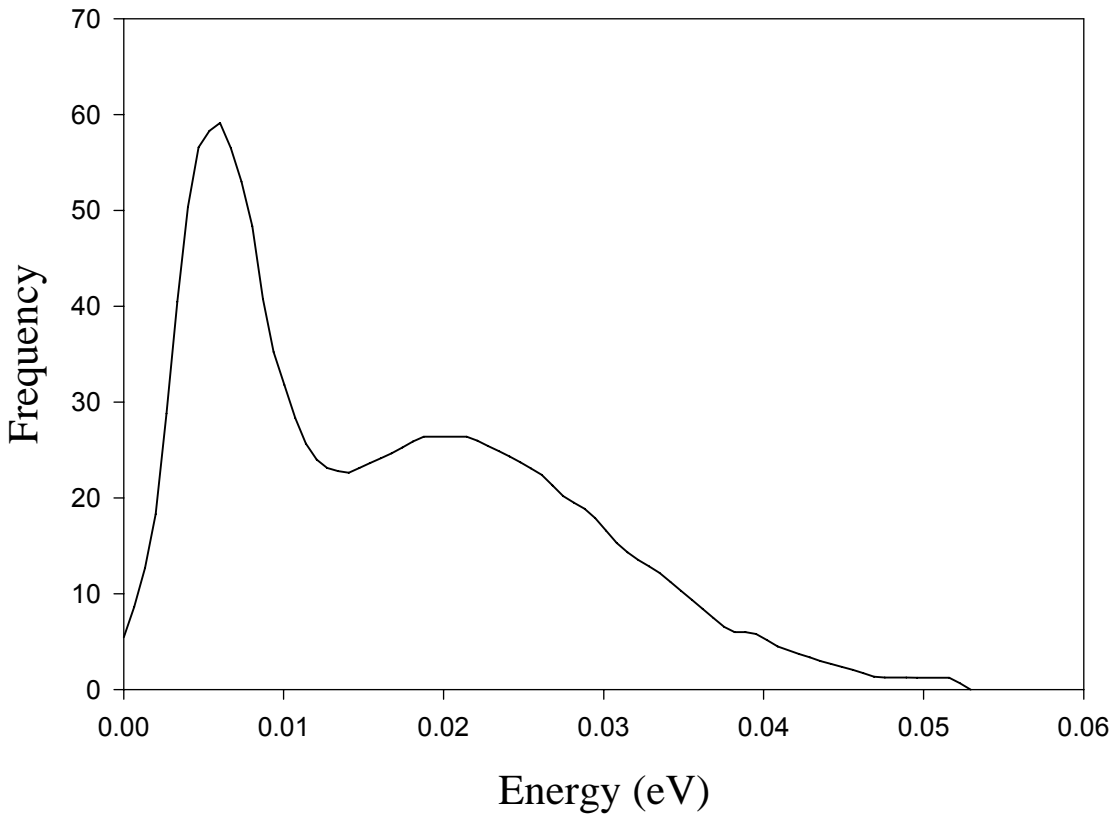

Fig. 14. The frequency spectrum of $\mathrm{O}$ in $\mathrm{H}_{2} \mathrm{O}$. 


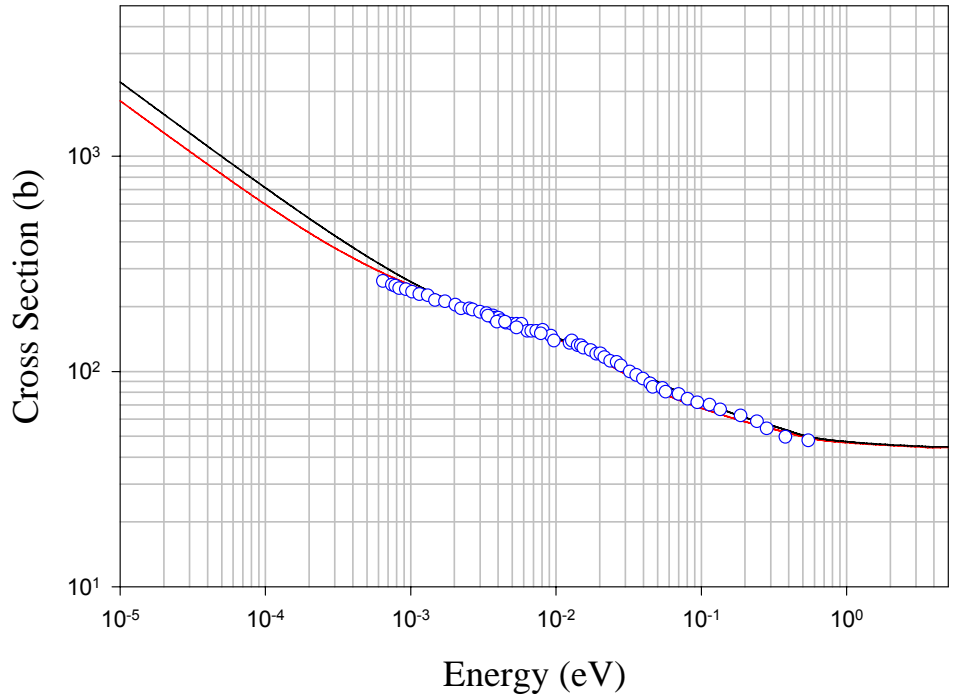

Fig. 15. The thermal neutron scattering cross section of $\mathrm{H}_{2} \mathrm{O}$ at 296 K. Calculated data are shown as solid lines (black for LEPAR data and red for the Edura-Morishima [24] model). BNL-325 [15] data are shown as blue circles.

The $\mathrm{BeO}$ dispersion relations have been calculated using a 2x2x2 supercell, 3x3x2 k-mesh, and $400 \mathrm{eV}$ Energy cut-off using the GGA approximation (Fig. 16). The calculated partial phonon spectra of Be and $\mathrm{O}$ in $\mathrm{BeO}$ are shown in Fig. 17 below.
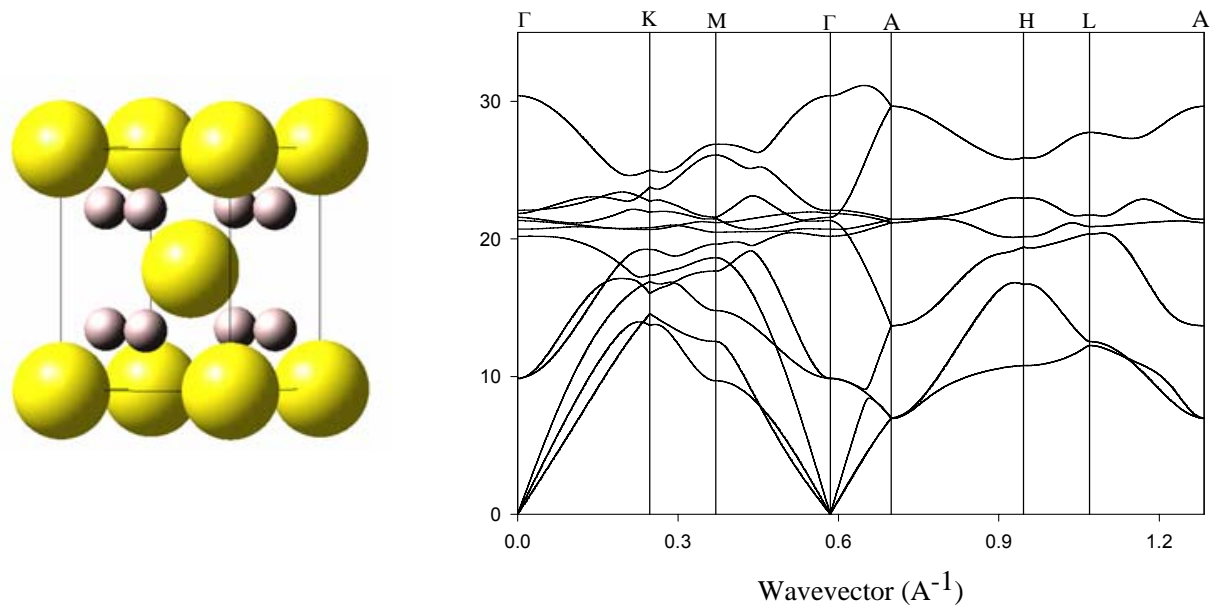

Fig. 16. The BeO unit cell (left) and the calculated dispersion relations (right). 

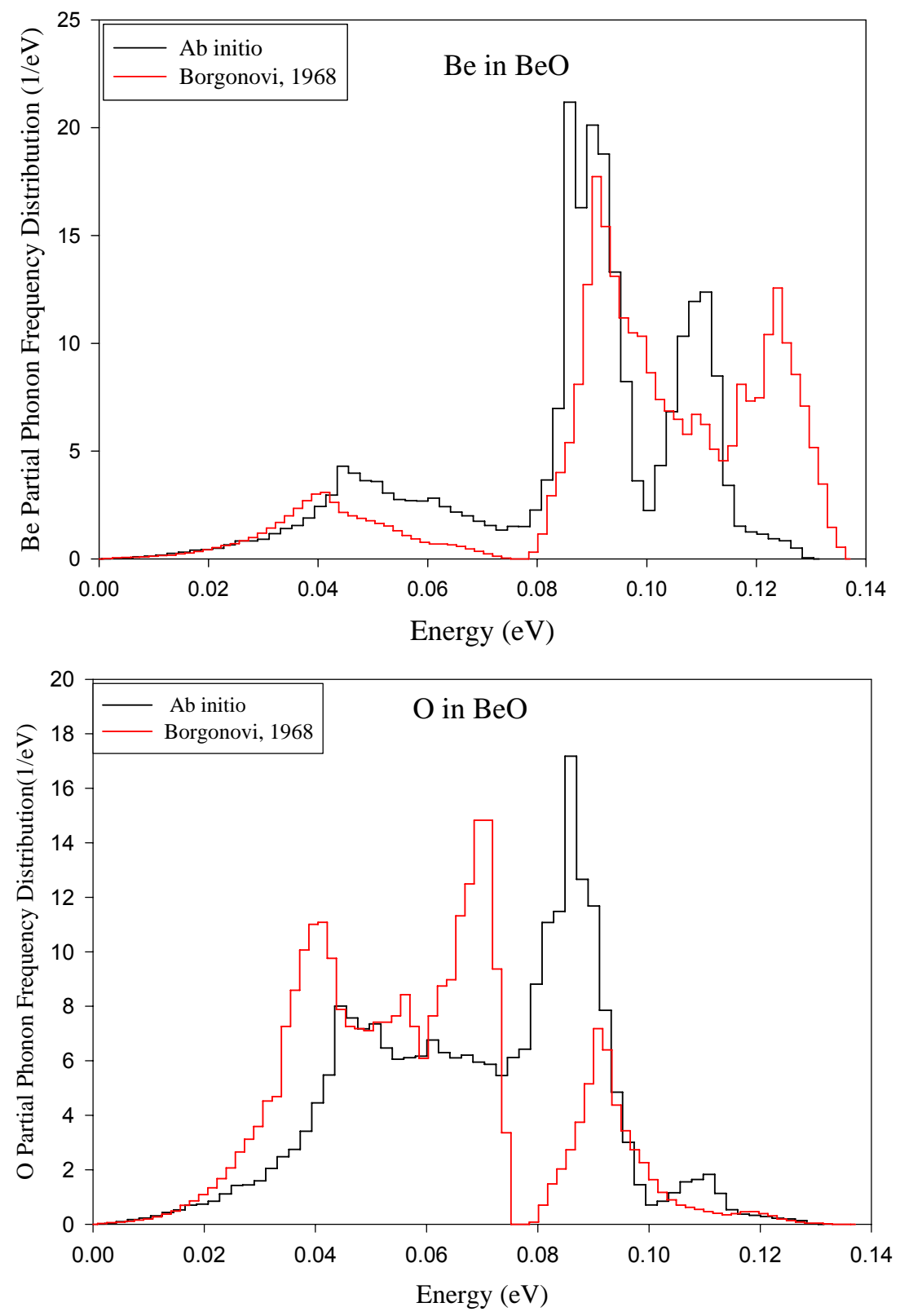

Fig. 17. The partial phonon spectra for Be and $\mathrm{O}$ in $\mathrm{BeO}$.

Figure 18 shows the thermal neutron scattering cross sections as generated by the NJOY/LEAPR code system. As it can be seen, the cross sections based on the ab initio analysis tend to be lower than the ones used in the common libraries $[15,25]$. 


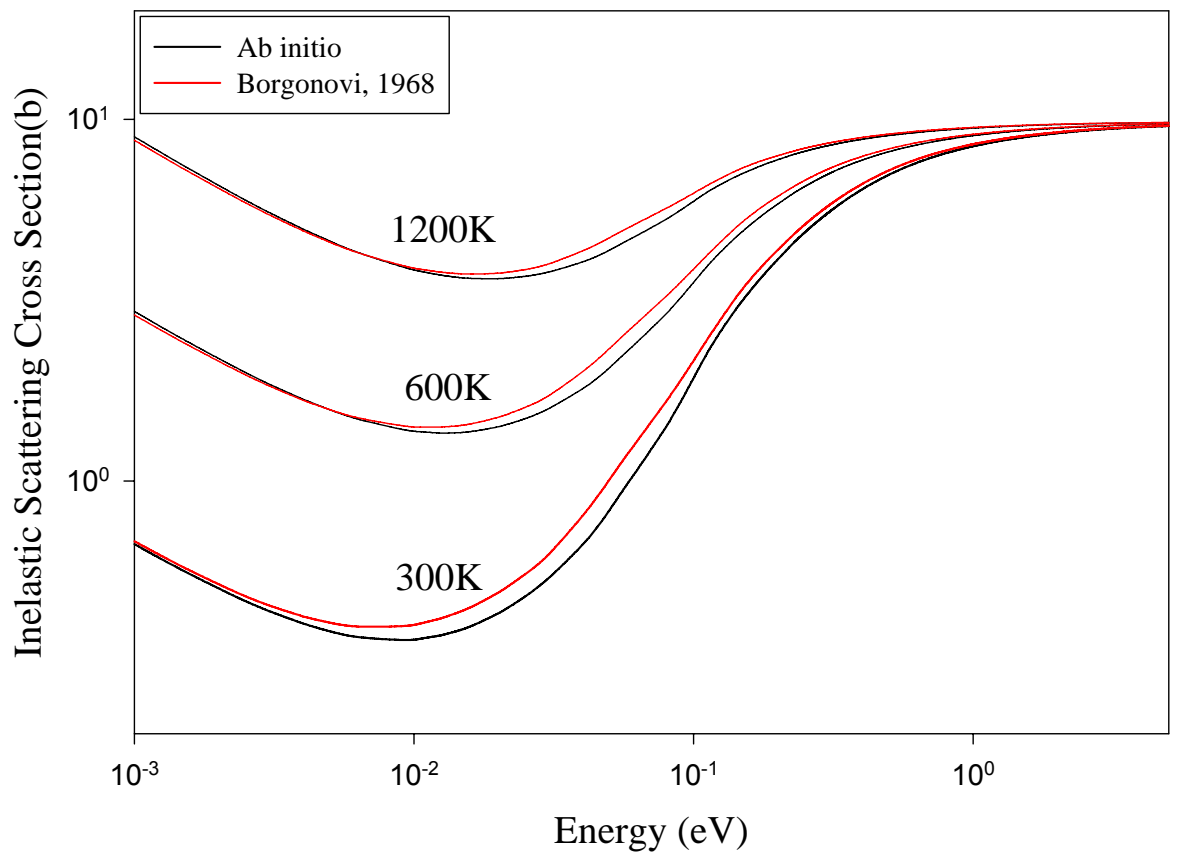

Fig. 18. The BeO thermal neutron inelastic scattering cross sections at various temperatures.

\subsection{Polyethylene $\left(\mathrm{CH}_{2}\right)$}

The inelastic thermal neutron scattering cross sections of polyethylene were updated. Figure 19 shows two density-of-states distributions for $\mathrm{CH}_{2}$. The first one was developed originally by Lin and Keoing [26], the low energy part of this spectrum was modified by Sprevak and Koppel [27] and implemented in GASKET, the red curve in the figure below, shows the density of states of $\mathrm{CH}_{2}$ that was used in GASKET (LKSK). The second density of states is by Lin and Keoing, but the low energy part was calculated by Swaminathan and Tewari [28]. Figure 20 shows the inelastic scattering cross sections based on the two phonon spectra generated using the LEAPR/NJOY system.

\section{Computational Benchmarks}

\subsection{Non-reactor Benchmarks}

The Be experiment described in Ref. [29] was utilized as a benchmark. In this experiment, the neutron leakage energy spectrum was measured for different reentrant-channel depths in a Be assembly; this provides a good 
check of the $\mathrm{Be}$ moderation and thermalization properties. The $\mathrm{Be}$ thermal scattering libraries that included the contribution of one-phonon coherent inelastic scattering cross sections were implemented and tested. MCNP5 models were run using these libraries. Figure 21 shows the results for the $30.5 \mathrm{~cm}$ reentrant channel. As it can be seen, accounting for the 1-phonon component results in significant enhancement for the agreement with the experimental data below $0.006 \mathrm{eV}$.

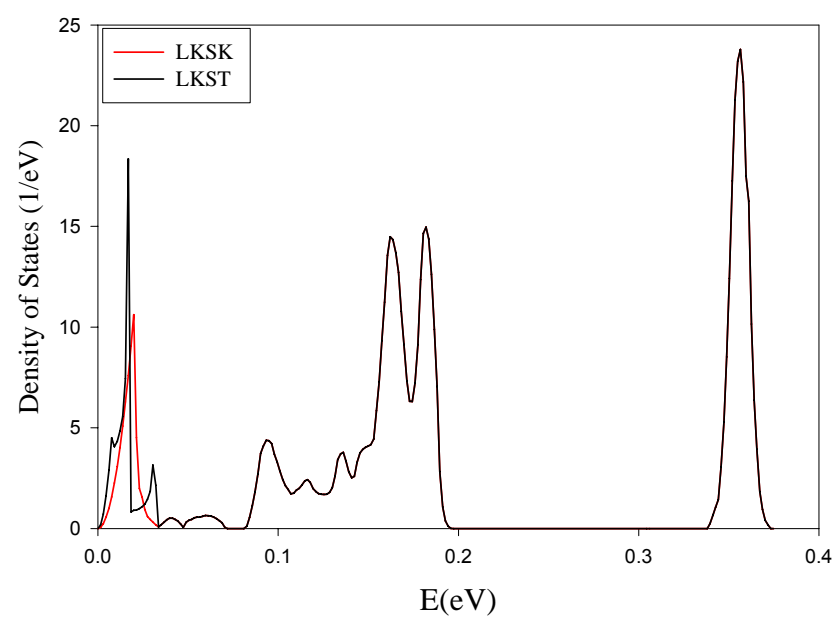

Fig. 19. The phonon density of States of $\mathrm{CH}_{2}$.

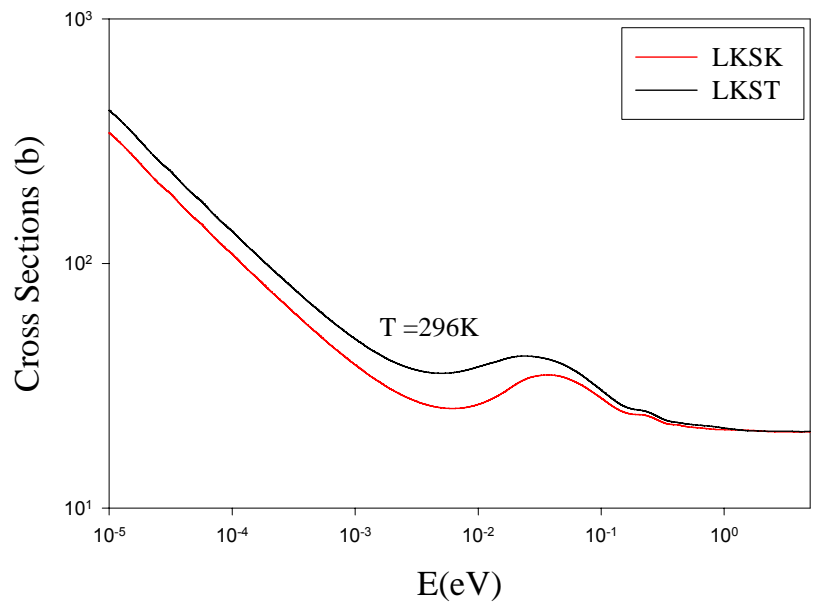

Fig. 20. The inelastic thermal neutron scattering cross section of $\mathrm{CH}_{2}$. 


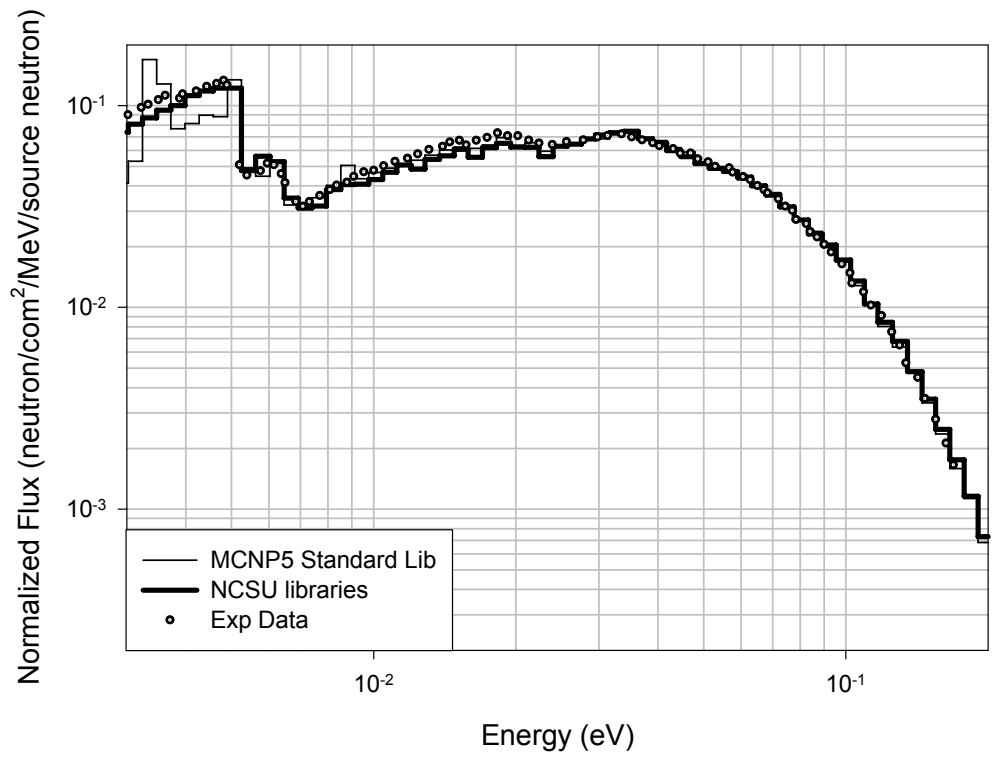

Fig 21. The leakage spectrum for the $30.5 \mathrm{~cm}$ depth reentrant channel in a $35.6 \times 35.6 \times 50.8 \mathrm{~cm}$ Be assembly. The NCSU Be cross-section libraries that include the contribution of one-phonon coherent inelastic scattering and fine meshes for initial and final energies were used in the calculation.

In the case of $\mathrm{BeO}, \mathrm{MCNP}$ simulations were performed for the $\mathrm{BeO}$ experimental benchmark as described in Ref. [30]. This experiment describes the neutron leakage energy distribution as a function of time from a $\mathrm{BeO}$ sample, which provides a good check of the $\mathrm{BeO}$ moderation and thermalization properties. Two libraries were used to calculate the neutron flux as a function of time and/or energy, and compared to the experimental data. The first is the Ab initio library which was generated by using the NJOY code with a phonon spectrum which was calculated by VSAP/PHONON, the other is the MCNP5 standard library. As shown in Figures 22 and 23 below, both libraries give good agreement with the experimental data.

In the case of light water, the experiment performed at General Atomics [31] was modeled using MCNP. In this experiment, the General Atomics electron linear accelerator and a tungsten alloy target, which was located beside the assembly, were used to produce neutrons. A bank of $\mathrm{BF}_{3}$ detectors was placed after a 16 meter collimator. A $10 \times 10 \times 10$ inches tank was filled with boric acid solution (effective absorption cross section is approximately 6 barns per hydrogen atom) or samarium nitrate solution (effective absorption cross section is approximately 9 barns per hydrogen 
atom). The experiment was performed at room temperature. The results of the benchmark (MCNP) simulations are shown in Figures 24 and 25. Both of the cross section libraries show reasonable agreement with the experimental data.
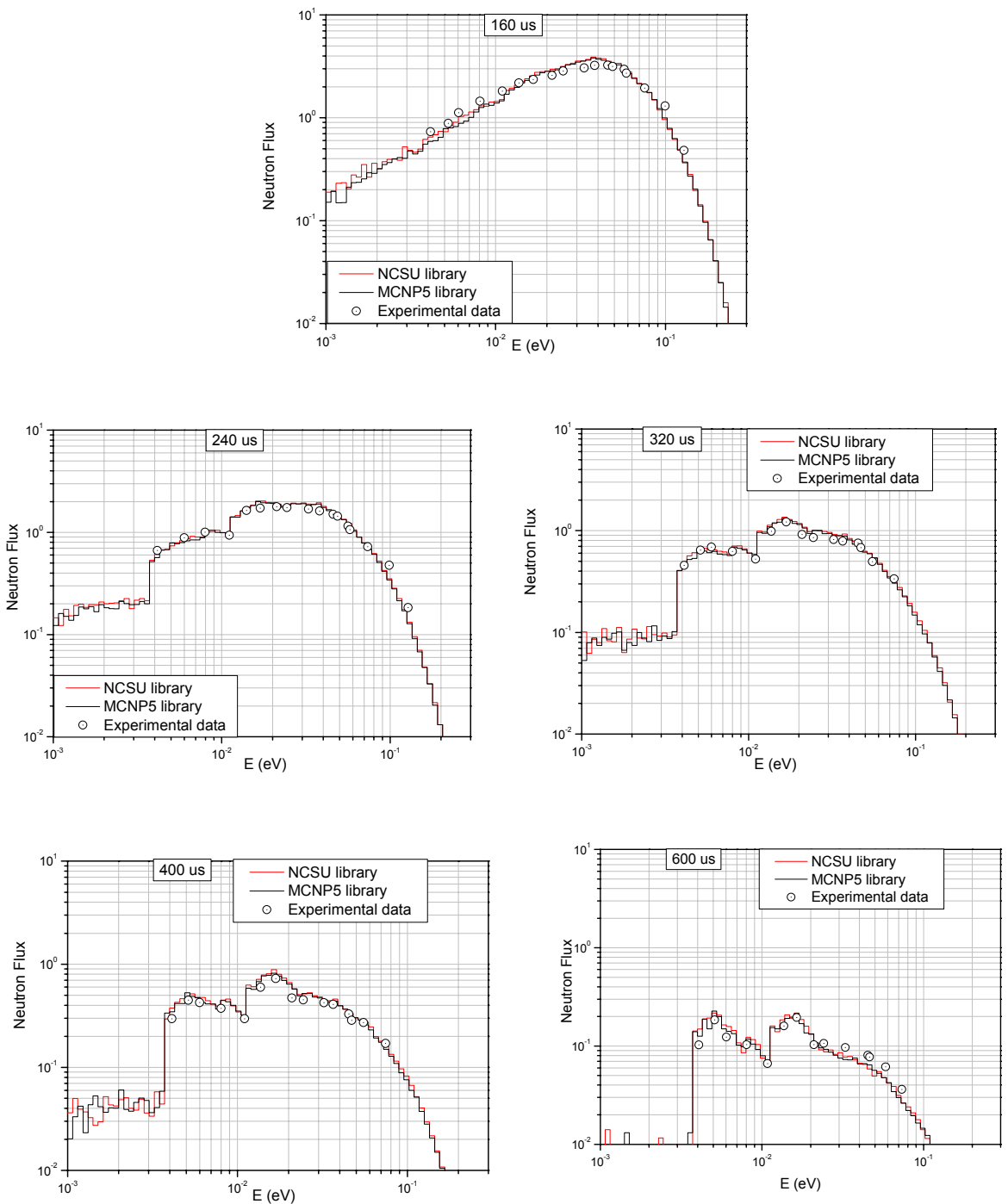

Fig 22. Time dependent leakage neutron energy spectrum for the $\mathrm{BeO}$ assembly. The agreement with experimental data is good for both the MCNP standard libraries and the NCSU ab initio libraries. 

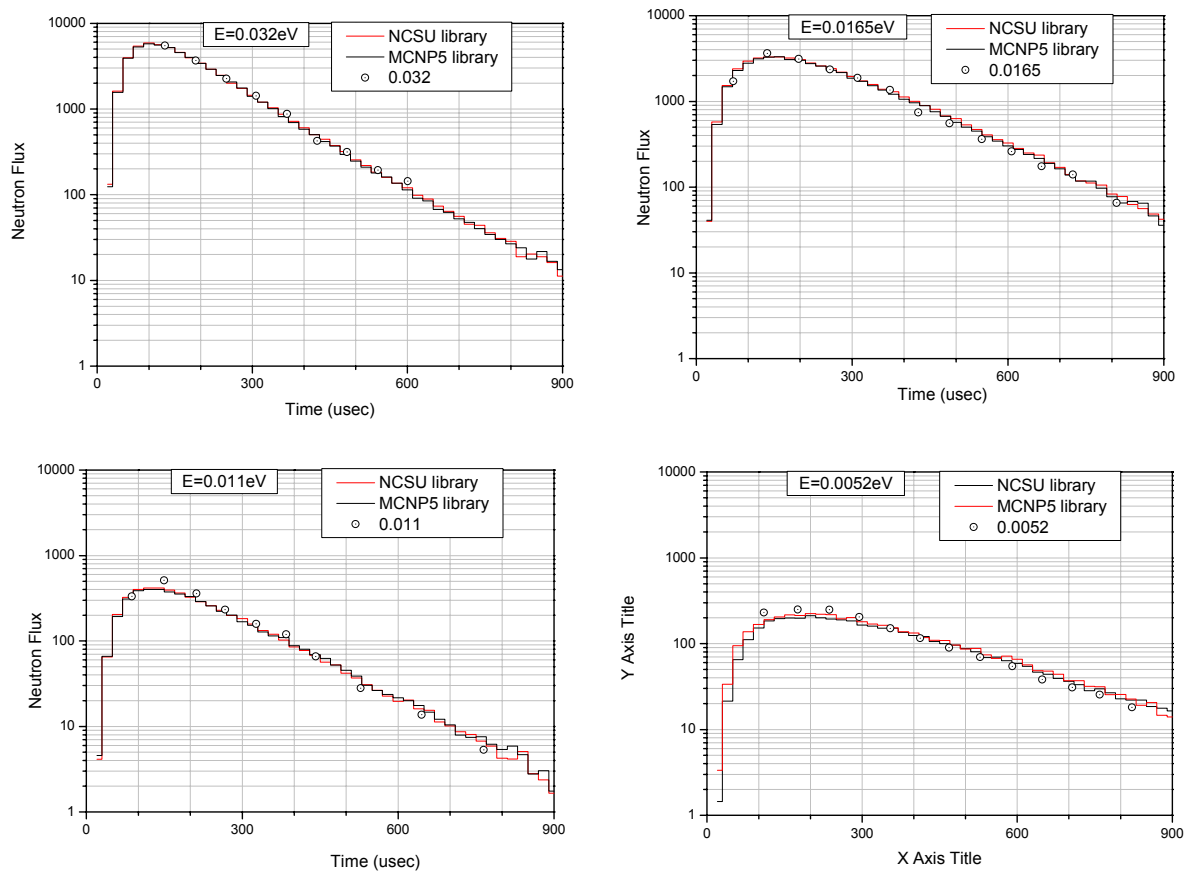

Fig 23. Energy dependent leakage neutron time spectrum for the $\mathrm{BeO}$ assembly. The agreement with experimental data is good for both the MCNP standard libraries and the NCSU ab initio libraries.

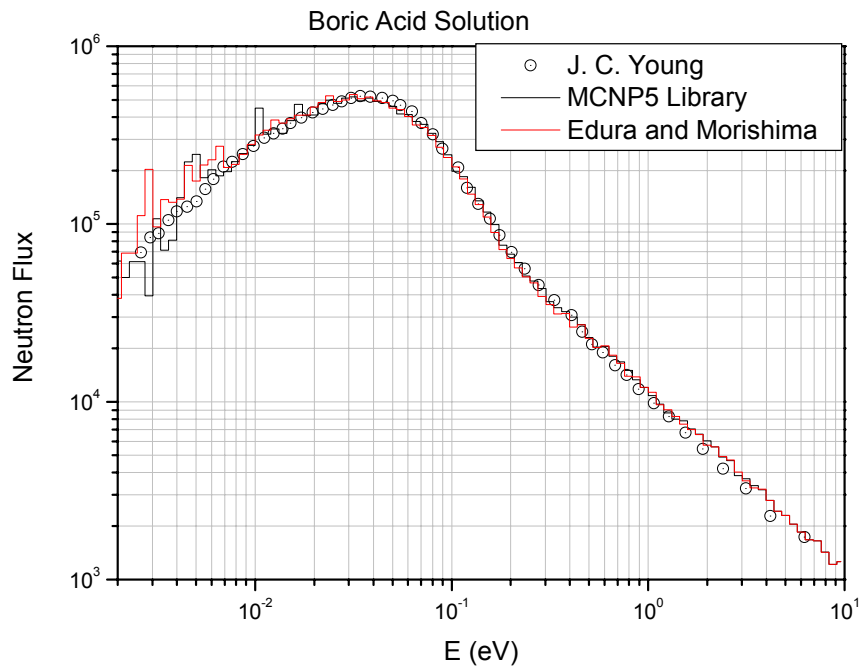

Fig. 24. Measured and calculated neutron spectra in boric acid solution. 


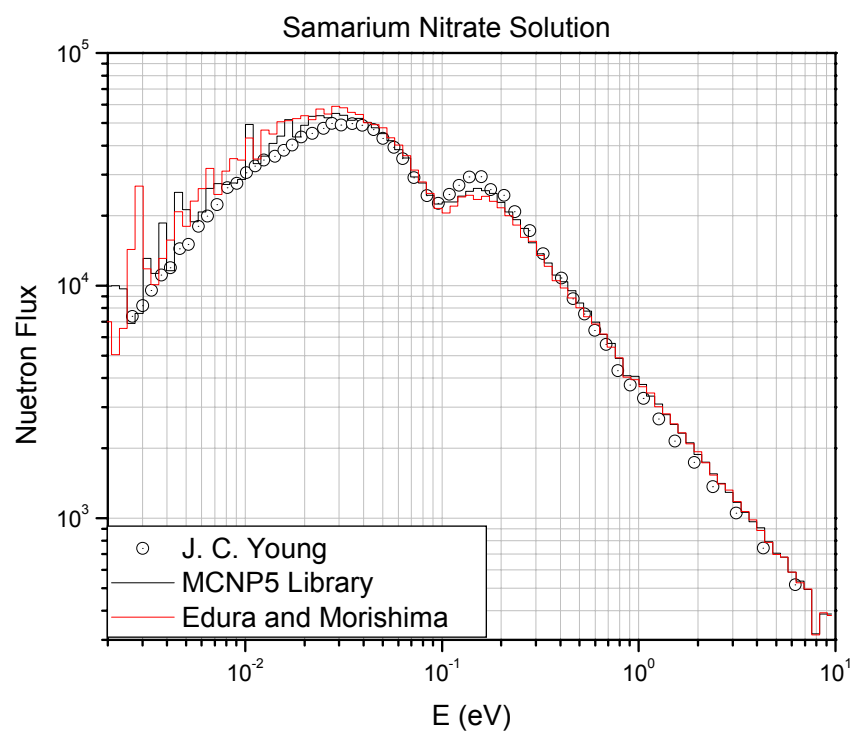

Fig. 25. Measured and calculated neutron spectra in samarium nitrate solution.

For $\mathrm{ZrH}$, the experiments described in [32] were used as the benchmark. The neutron spectra are measured through time-of-flight techniques. Figure 26 shows results of the benchmark. At $T=670 \mathrm{~K}$, the simulation predicts the temperature dependence of the $\mathrm{ZrH}$ optical modes with a peak around the scattered neutron energy of $0.137 \mathrm{eV}$. As it can be seen, good agreement between calculation and measurement is observed in this benchmark especially if the broad energy bins that are used in the experiment are also utilized in the simulation.

The other simulation involves equilibrium thermal neutron spectra measured in a $\mathrm{ZrH}$ cube $0.254 \mathrm{~m}$ on a side, which is filled with $\mathrm{ZrH}_{1.75}$ powder mixed with $\mathrm{B}_{4} \mathrm{C}$ particles at a concentration of 3.4 barns per hydrogen atom [33]. This benchmark calculation was performed at the University of Michigan using the NCSU cross sections. Neutron spectra are determined through time-of-flight measurements of neutrons exiting from the center of the cube through a copper guide tube, with diameter $D$ $=25.4 \mathrm{~mm}$ and length $L=0.127 \mathrm{~m}$. MCNP5 simulations were performed by tallying the neutron spectra at the center of the cube and accounting for neutron reflection along the tube walls. Figure 27 shows the comparison between the MCNP5 simulations and the experimental results. As it can be seen, the standard MCNP5 libraries and the NCSU libraries reproduce the experimental results well. 


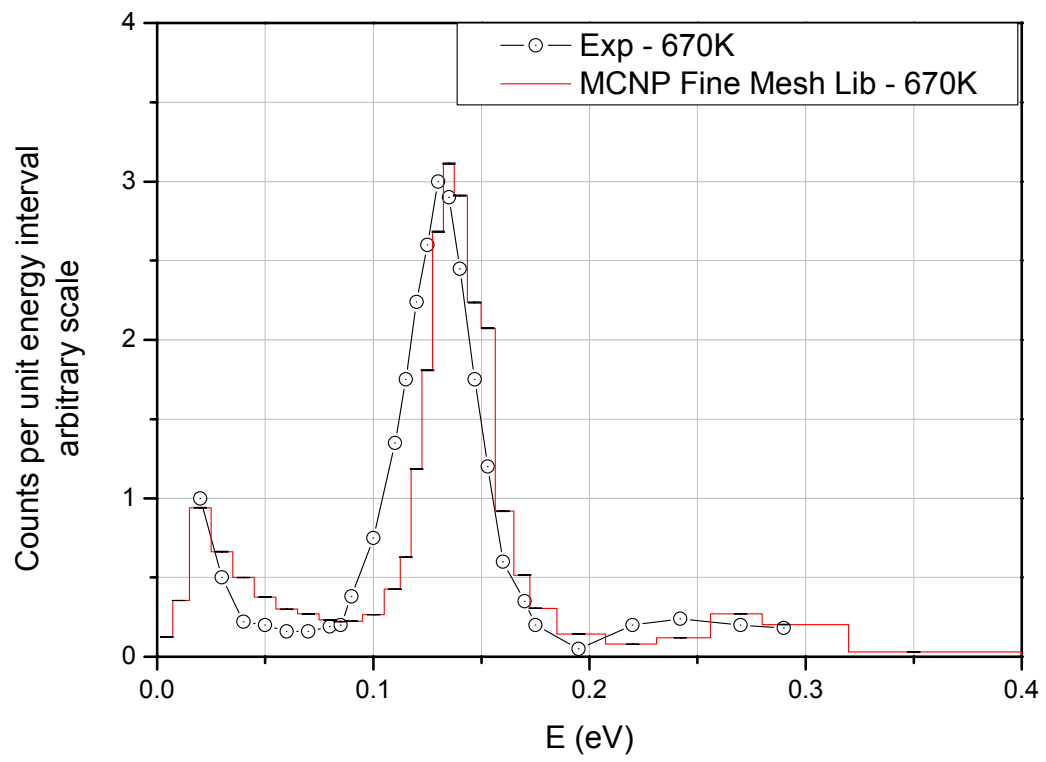

Fig. 26. Energy distribution of 4-meV neutrons scattered off a ZrH sample.

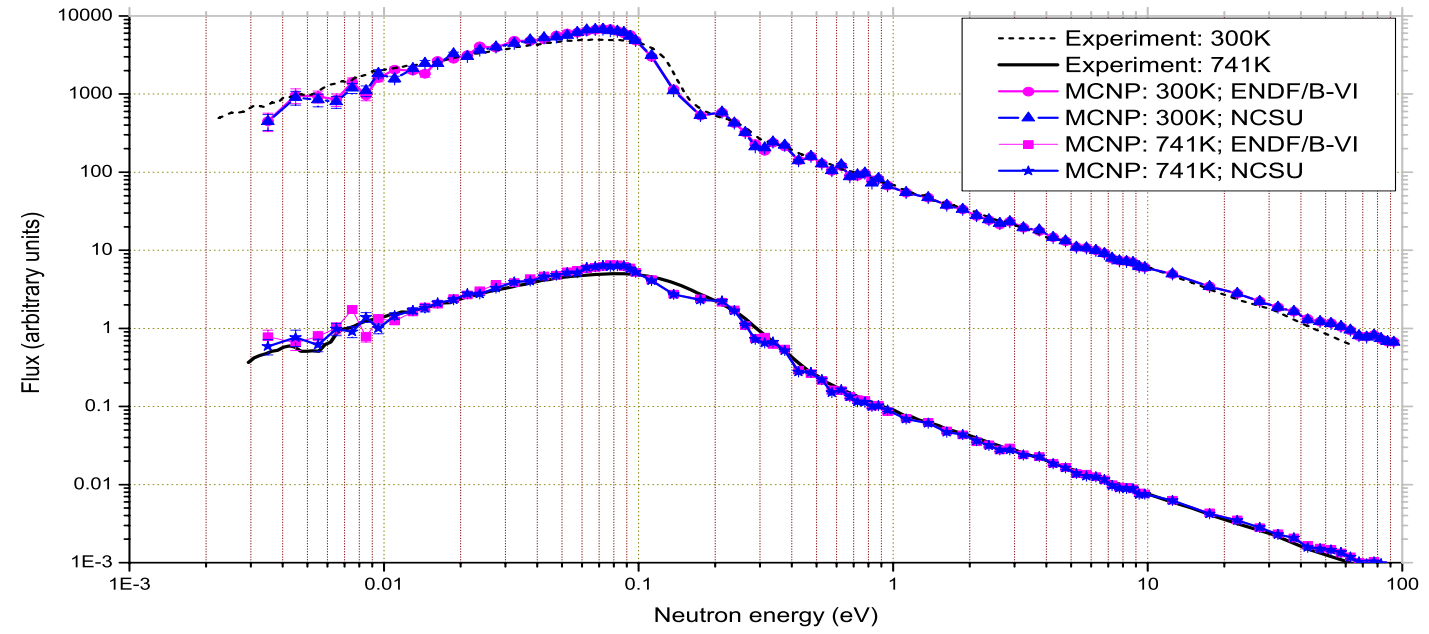

Fig. 27. Equilibrium neutron spectra in a ZrH block.

As it can be seen from the above results, the agreement between experimental and computational results is generally good for light water and $\mathrm{ZrH}$. However, this outcome should also be evaluated using any recently published analysis for these moderators/reflectors in multiplying and non-multiplying systems. 
For polyethylene, the experiment described in [34] was used as a benchmark. Reasonable agreement between simulations performed using the MCNP code (with ENDF/B-VI data) and the experimental data is found. The comparison is shown in Fig. 28 below.

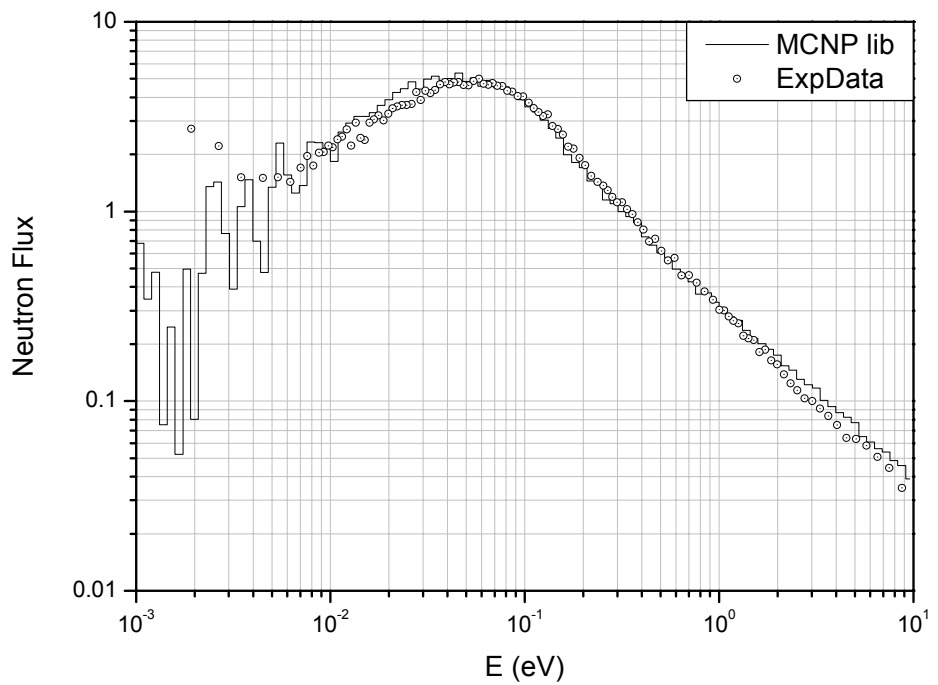

Fig 28. Simulated vs. experimental spectra for the poisoned polyethylene benchmark experiment.

\subsection{Reactor Benchmarks}

\section{CRITICAL EXPERIMENTS WITH Be and BeO}

The four cases analyzed are described in the report Two BeryliumModerated Crtical Assemblies by E.L. Zimmerman, ORNL-2201 (October 1958). For the two bare critical system described in this report we added the analysis of 2 more reflected systems built during the experiments. The report was recently reevaluated (document NEA/NSC/DOC(95)03/II Volume II HEU-MET-THERM-025). Highly enriched (93.4\%) U foils (2.86 in diameter, 10 mils thick) where sandwiched with 1 in thick-2.875 in side square Be blocks. The two moderation ratios, nominally $\mathrm{n}(\mathrm{Be}) / \mathrm{n}(\mathrm{U} 235)=390$ and 1560, were obtained by assembling, respectively, 1 foil per one Be block or 1 foil per four Be blocks; the bare assemblies were called CA-1 and CA-18. The reflected cases are going to be called the same name with the adjective reflected (they obviously have less fuel). All the cases were built in a honeycomb array of $24 \times 24$ Al square tubes 3 in outside diameter (total array $6 \times 6$ foot). The empty parts of the array were also modeled. 


\begin{tabular}{|l|l|l|l|l|}
\hline \multicolumn{1}{|c|}{ Case } & $\mathrm{nBe} / \mathrm{nU} 235$ & $\mathrm{k}$ measured & $\mathrm{k}$ ENDF-B/VI & k ENDF-B/V \\
\hline CA-1 Bare & 390 & 1.0054 & $1.0232(7)$ & -- \\
\hline CA-1 Reflected & 390 & $\sim 1.0054$ & $1.0276(7)$ & -- \\
\hline CA-18 Bare & 1560 & 1.0020 & $1.0128(8)$ & $0.9991(8)$ \\
\hline CA-18 Reflected & 1560 & $\sim 1.0020$ & $1.0143(7)$ & $0.9997(7)$ \\
\hline
\end{tabular}

Some sensitivity analyses were done for the case of the less moderated assembly with respect to the effective number density of Be. At this moderation there is a 1 to 1 sensitivity of $k$ to $n(B e):-1 \%$ change in $n(B e)$ produces $-1 \%$ change in $\mathrm{k}$. The original report only gives the density of $\mathrm{Be}$ $(1.86 \mathrm{~g} / \mathrm{cc})$. The evaluators of this work assigned a $1 \%$ impurity in $\mathrm{Be}$ with an isotopics that reduces $\mathrm{n}(\mathrm{Be})$ slightly more than $1 \%$. Therefore the bias in this case $+1.78 \% \mathrm{k}$ might depend on this assumption.

Furthermore, the 14 "clean" configurations (bare critical parallelepiped) calculated with MCNP are described in the article Critical Dimensions of Unreflected BeO Systems Fueled by Thin Enriched Uranium by F.A. Kloverstrom, R.M. Dek and A.J. Reyenga (UCRL-L) published in TANS, Vol 2, page 162(1959). In these highly enriched assemblies $U$ foils (93.2 $\%$ enriched, 1,2,3,4,6 and 8 mils thick, square and triangular in shape) were assembled between slabs of $\mathrm{BeO} 1,2,3$ and 4 inches wide. The geometry data available are the dimension of the parallelepipeds and details in the axial direction but not in the horizontal plane (the plane of the $U$ foils), so we have to make some assumptions that are related to the leakage in the horizontal direction and the shielding in the foils. The material information available is the density of the $\mathrm{BeO}$ and the ratio between the average number densities of $\mathrm{BeO}$ and U235. The density and the extension of the foils in $(x, y)$ were not published.

The extension of the $U$ foils in the horizontal plane is smaller than the extension of the moderator. Because there is no information about this, we started with the assumption of a uniform $(x, y)$ distribution to find an effective uranium density that would make possible to infer the void between foils. The uranium density is given by

$\rho_{U}=\left(\frac{V_{B e O}}{V_{U}}\right)\left(\frac{A_{U}}{A_{B e O}}\right)\left(\frac{n_{U 235}}{n_{B e}}\right) \frac{1}{e n r} \rho_{B e O}$

The first parenthesis is the ratio of the volumes of $\mathrm{BeO}$ and the $U$ foils, the second is the ratio of atomic numbers, the third is the ratio of number densities; the other two terms are the enrichment and the $\mathrm{BeO}$ density. In this formula all is known except the first parenthesis. As a starting hypothesis we assumed a uniform distribution of $U$ in the horizontal plane 
so the parenthesis is given by the ratio of thicknesses (known). The input data and the results of the calculations are given in Tables 1 and 2

The yellow fields in Table 1 are original or other data needed in the modeling, the assumed $U$ foils density (oraloy) was taken from the literature of that time; it is used in Table 2 to deduce the voids between foils

Table 1. Input Data for the $14 \mathrm{BeO}$ configurations Critical Size of Parallelepipeds

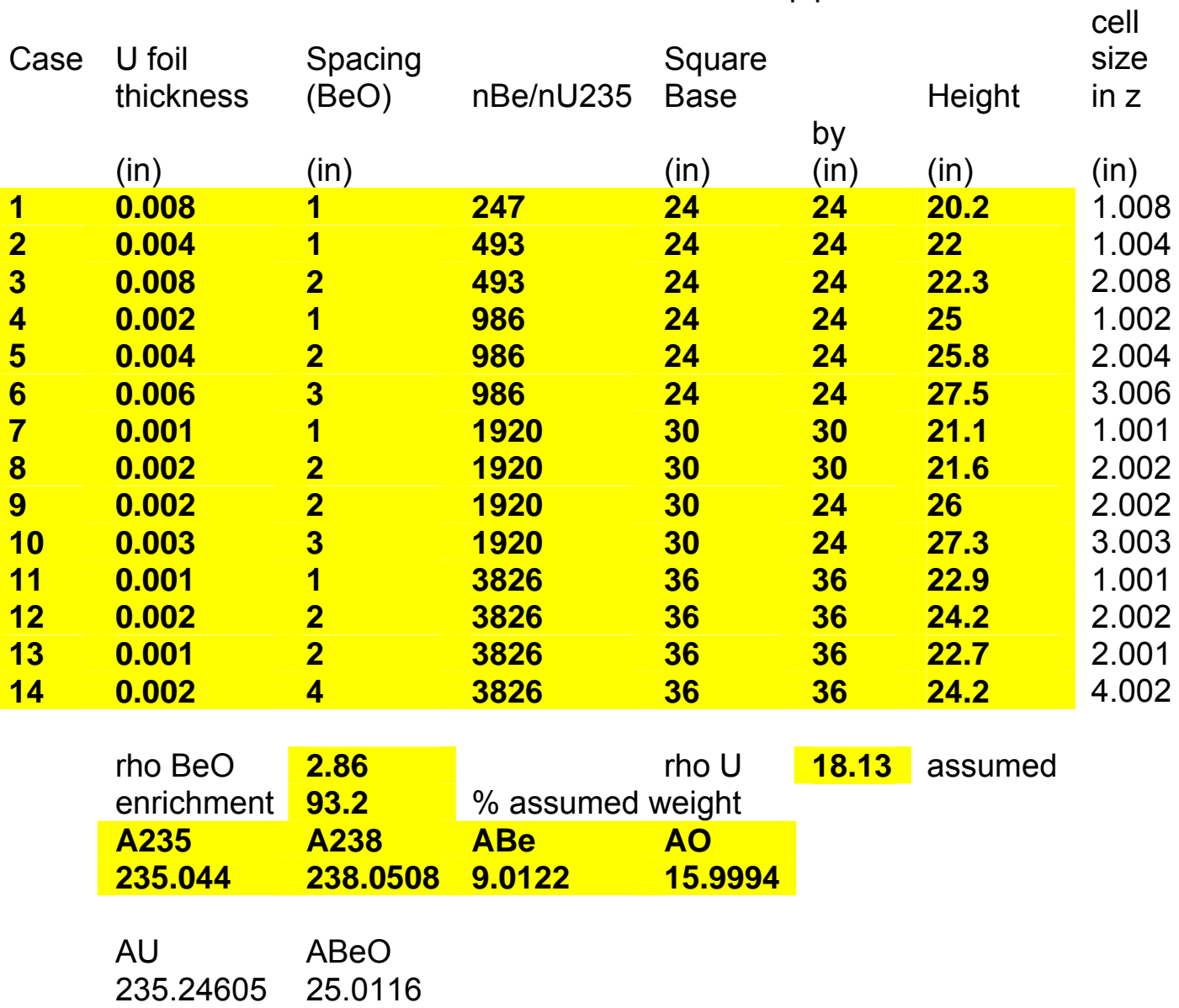

Table 2 summarizes the results for the average density of the foils, given by Eq. 1, the multiplication of each assembly and the biases of the calculations (for 200,000 histories and 2 million histories). The values in parenthesis are the error of the last digit of the k's. With the assumed $U$ density of table 1 , the effective area in $(x, y)$ occupied by the foils can be 
inferred; larger than 0.8 for the case of the square foils and around 0.42 for the case of the triangular foils. With these numbers, and the relations between the thickness of the foil and the $\mathrm{BeO}$ it is possible to calculate the void per unit area, beta, for each one of the four lateral sides. The biases of the calculations are in general less than $1 \%$ except in the case of the triangular foils which are more diluted, in the models, than the other cases. This would justify second iterations of calculations without the uniform $(x, y)$ hypothesis.

Table 2. Results for the $14 \mathrm{BeO}$ Configurations

\begin{tabular}{|c|c|c|c|c|c|}
\hline \multicolumn{3}{|c|}{ "uniform(xy)"rho } & \multicolumn{3}{|c|}{$x y$} \\
\hline & $\mathrm{U}$ & $200 \mathrm{KH}$ & $2 \mathrm{MH}$ & Udilution & \\
\hline case & $\mathrm{g} / \mathrm{cc}$ & k MCNP & k MCNP & $<$ rho $>$ /rho & Ufoils \\
\hline 1 & 14.723 & $1.007(2)$ & $1.0065(8)$ & 0.812095 & square \\
\hline 2 & 14.695 & $1.008(2)$ & & 0.810513 & " \\
\hline 3 & 14.695 & $1.010(2)$ & $1.0073(8)$ & 0.810513 & $"$ \\
\hline 4 & 14.665 & $1.009(2)$ & & 0.808898 & $"$ \\
\hline 5 & 14.665 & $1.001(3)$ & & 0.808898 & $"$ \\
\hline 6 & 14.665 & $1.013(2)$ & $1.0132(8)$ & 0.808898 & $"$ \\
\hline 7 & 15.047 & $1.003(3)$ & & 0.829977 & $"$ \\
\hline 8 & 15.047 & $0.999(2)$ & & 0.829977 & " \\
\hline 9 & 15.047 & $1.006(2)$ & & 0.829977 & $"$ \\
\hline 10 & 15.047 & $1.006(2)$ & $1.0078(8)$ & 0.829977 & " \\
\hline 11 & 7.551 & $1.026(2)$ & & 0.416507 & triangular \\
\hline 12 & 7.551 & $1.043(2)$ & $1.0459(7)$ & 0.416507 & ""' \\
\hline 13 & 15.095 & $1.008(2)$ & & 0.832598 & square \\
\hline 14 & 15.095 & $1.007(2)$ & $1.0057(7)$ & 0.832598 & \\
\hline case & nBe/NU235 & $\begin{array}{c}200 \mathrm{KH} \\
\mathrm{dk}(\%)\end{array}$ & $\begin{array}{c}2 \mathrm{MH} \\
\mathrm{dk}(\%)\end{array}$ & beta= & \\
\hline 1 & 247 & 0.7 & 0.65 & 0.000784 & \\
\hline 2 & 493 & 0.8 & & 0.000397 & \\
\hline 3 & 493 & 1 & 0.73 & 0.000397 & \\
\hline 4 & 986 & 0.9 & & 0.000201 & \\
\hline 5 & 986 & 0.1 & & 0.000201 & \\
\hline 6 & 986 & 1.3 & 1.32 & 0.000201 & \\
\hline 7 & 1920 & 0.3 & & 0.000089 & \\
\hline 8 & 1920 & -0.1 & & 0.000089 & \\
\hline 9 & 1920 & 0.6 & & 0.000089 & \\
\hline 10 & 1920 & 0.6 & 0.78 & 0.000089 & \\
\hline 11 & 3826 & 2.6 & & 0.000354 & \\
\hline 12 & 3826 & 4.3 & 4.6 & 0.000354 & \\
\hline 13 & 3826 & 0.8 & & 0.000044 & \\
\hline 14 & 3826 & 0.7 & 0.57 & 0.000044 & \\
\hline
\end{tabular}




\section{Graphite Experimental Measurements and Benchmarks}

\subsection{Total Cross Section Measurements}

Experiments were performed at the National Institute of Standards and Technology (NIST) to measure the total cross section of the interaction of neutrons that have a wavelength of $0.89 \mathrm{~nm}$ (i.e., $\sim 1 \mathrm{meV}$ ) with various types of graphite. The energy for these neutrons falls below the Bragg cut-off for graphite $(\sim 2 \mathrm{meV})$. Therefore, the total cross section will generally be due to inelastic neutron scattering and absorption interactions. In the case of carbon (i.e., graphite), absorption represents a minor contribution. Consequently, this measurement yields a direct estimate of the inelastic scattering cross section of graphite for neutrons with sub-Bragg energies. Clearly, this is a complementary experiment to the slowing-down-time experiment that was setup and performed at the ORELA facility of ORNL where inelastic neutron scattering above the Bragg cut-off will be probed.

The experiment at NIST was performed on neutron guide \#6 (NG-6) and at room temperature. Figure 29 below shows the experimental setup. A monochromator composed of nine tiled pieces of stage 2 potassium intercalated graphite was used to extract the $1 \mathrm{meV}$ beam. Other higher order contaminations also existed in the beam. Filtering of the higher order contamination was achieved using a highly oriented pyrolitic graphite filter. The resulting neutron spectrum was measured using the time-of-flight (TOF) method. Figure 30 shows the measured neutron spectrum that was used to perform the transmission measurements. Approximately $90 \%$ of the neutrons in the spectrum reside in the $\lambda=0.89$ $\mathrm{nm}$ wavelength peak, which corresponds to an energy of $1 \mathrm{meV}$.

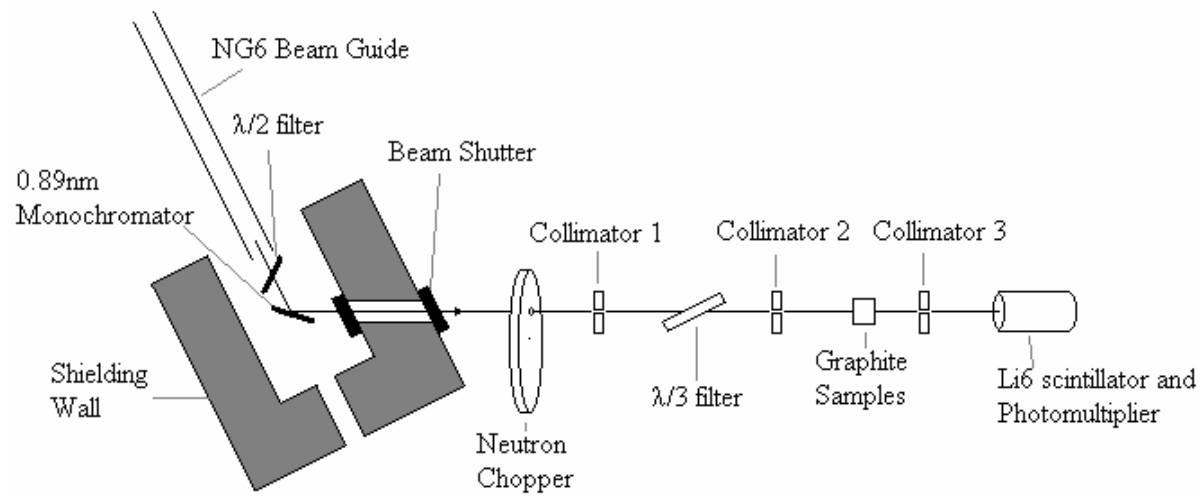

Fig. 29. The experimental setup for the measurement of the graphite total cross section on NG \#6 at NIST. 


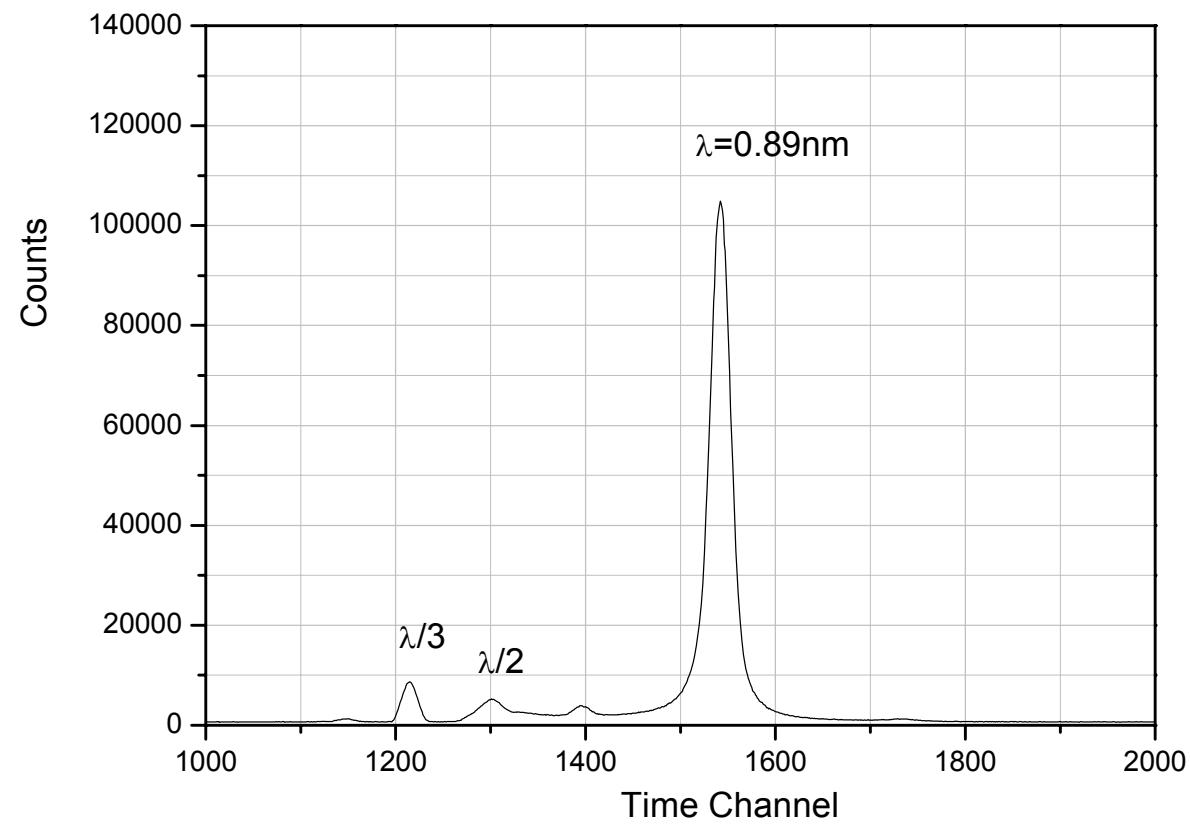

Fig. 30. The neutron spectrum after filtration and extraction using a monochromator as shown in Fig. 27. $\lambda=0.89 \mathrm{~nm}$ corresponds to $1 \mathrm{meV}$ neutrons.

As a first step, to confirm our experimental approach and procedures, the experimental method to perform the total cross section measurement was tested using aluminum samples (99.9\% pure). Due to beam intensity considerations, the measurements were performed using the continuous beam and not in time-of-flight (TOF) mode. However, the spectral data obtained from the TOF mode (Fig. 30) was used to correct the measurement and obtain the value of the total aluminum cross section at $1 \mathrm{meV}$. Figure 31 shows the result of decomposing the spectrum into its different components. Figure 32 shows the transmission curve for the aluminum samples. The curve shows that the measured total cross section for aluminum (at $1 \mathrm{meV}$ ) in this experiment is $\sim 1.4$ barns, which is in good agreement with the value of 1.44 barns as reported in $\mathbf{D}$. $\mathbf{J}$. Hughes and R. B. Schwartz, "Neutron Cross Section," BNL 325, Brookhaven National Laboratory [15]. The 3\% difference between the two values is considered to be within the uncertainty limits of the experiment. 


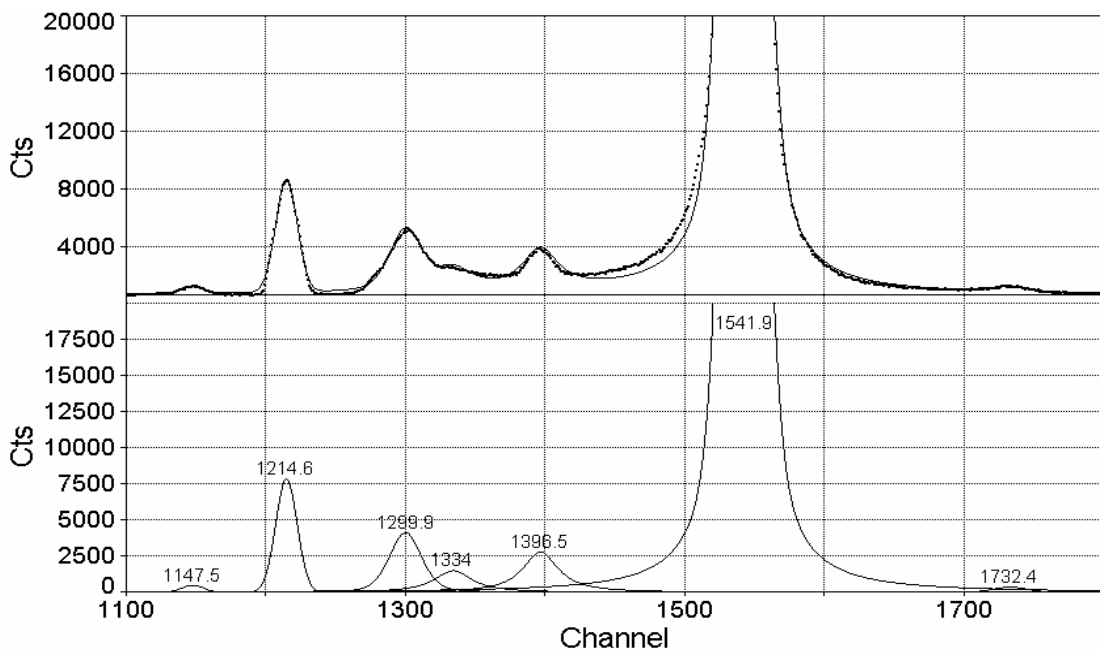

Fig. 31. The various wavelength (i.e., energy) components of the TOF spectrum shown in Fig. 28.

Based on the above, identical procedures were applied to establish the transmission curves of $0.89 \mathrm{meV}$ neutrons through samples of reactor grade and pyrolitic graphite. Figures 33 and 34 below show the results. At this stage, the values of the measured total cross section are $0.43 \pm 0.05$ barns for pyrolitic graphite and $0.61 \pm 0.05$ barns for reactor grade graphite. For pyrolitic graphite this value is in reasonable agreement with the value of 0.37 barns that is inferred in A. Steyerl and W. D. Trustedt, "Experiments with a Neutron Bottle," Z. Physik, 267, 379-388 (1974) [5]. For reactor grade graphite, the measured value is in reasonable agreement with the value of 0.66 barns as reported in $\mathbf{D}$. $\mathbf{J}$. Hughes and R. B. Schwartz, "Neutron Cross Section," BNL 325, Brookhaven National Laboratory [15].

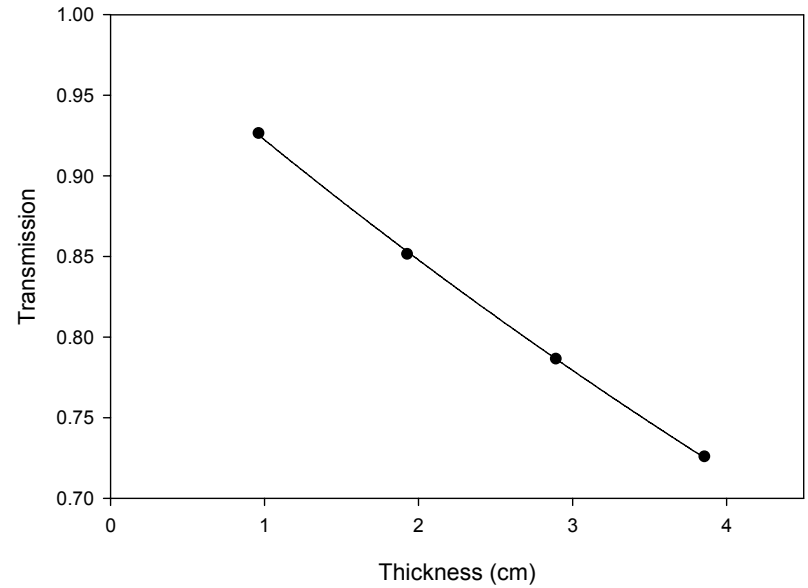

Fig. 32. The measured transmission curve of $1 \mathrm{meV}$ neutrons through aluminum. 
Final Project Report

DE-FG07-02SF22514

June 20, 2008

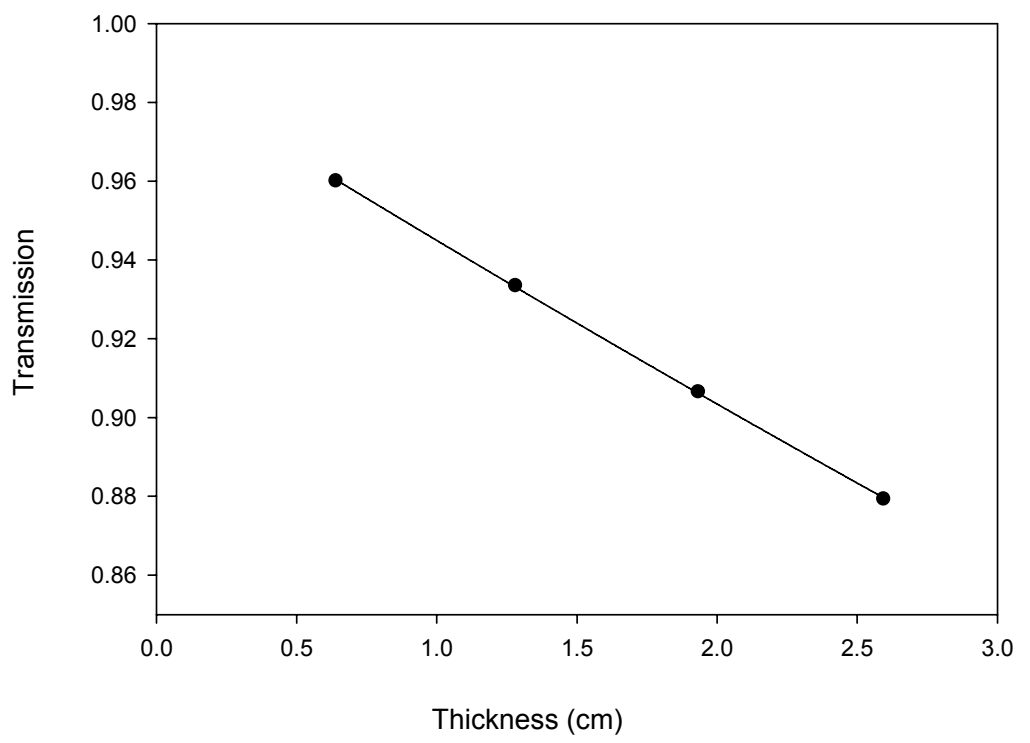

Fig. 33. The measured transmission curve of $1 \mathrm{meV}$ neutrons through pyrolitic graphite.

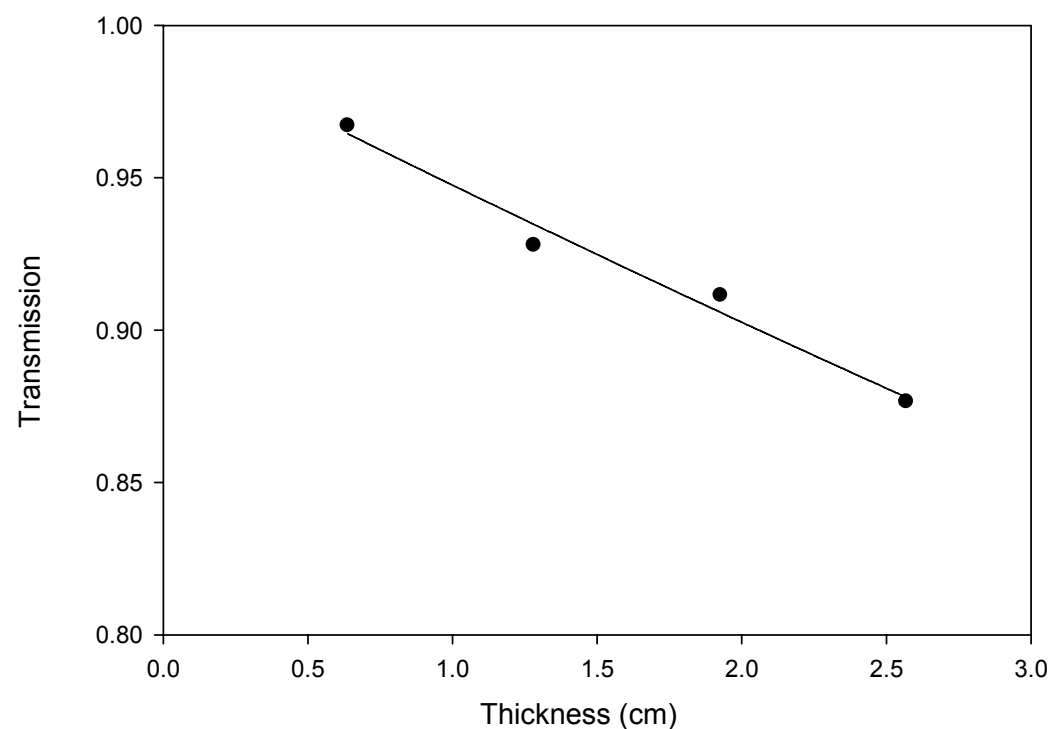

Fig. 34. The measured transmission curve of $1 \mathrm{meV}$ neutrons through reactor grade graphite. 


\subsection{Slowing-Down-Time Measurements}

\section{Basic Concept and setup}

The neutron slowing-down-time technique is based on the fundamental coupling between the energy of a neutron as it slows down in a moderator and the time it is required to reach that energy. This technique was previously used to measure reaction cross sections of a given material (e.g., the fission cross section of $U-238$ ) that is placed within an appropriate moderating structure (e.g., Pb was the preferred moderator of choice). The work in this project represents significant deviation from past implementations of the slowing-down-time concept. Specifically, this work represents the first attempt to use this concept as an integral benchmark technique for understanding the interactions of neutrons within the moderator and in this case thermalization behavior. Furthermore, the detection of time dependent signal in this experiment is performed using detectors placed outside the moderating structure, which is based on assuming that the neutron time-energy coupling is preserved for the leaking neutron field as it is for the field internal to the structure. In this work, this would allow for enclosing the moderating structure within a custom designed heater, which would enable temperature dependent slowing-down-time experiments in graphite.

Based on the concept presented above, and using extensive Monte Carlo (MCNP) simulations the size of the graphite pile for the experiment (at several temperatures) was defined to be a $70 \times 70 \times 70 \mathrm{~cm}^{3}$ cube. This allowed us to preserve the main characteristics of the system as a spectrometer, while minimizing the power needed to heat the system, and using most efficiently the graphite that we have. It is also possible to measure at room temperature two additional piles that are $50 \times 50 \times 50 \mathrm{~cm}^{3}$ and $100 \times 100 \times 100 \mathrm{~cm}^{3}$ to check the quality of the simulations with samples of different buckling. In addition, at room temperature experiments can be performed with the detector in the center of the pile, to minimize the effects of room return.

The heating system to be used in the experiments has been designed, constructed and is currently set up at the ORELA facility of ORNL. The design and optimization of the heating system accounted for neutron room return effects and the shielding was designed to minimize this effect. Fig. 35 shows a schematic of the system design. The set up of the experiment and all its associated systems was executed in full coordination wit the to the ORELA staff to ensure compliance with the required safety and operational standards. 


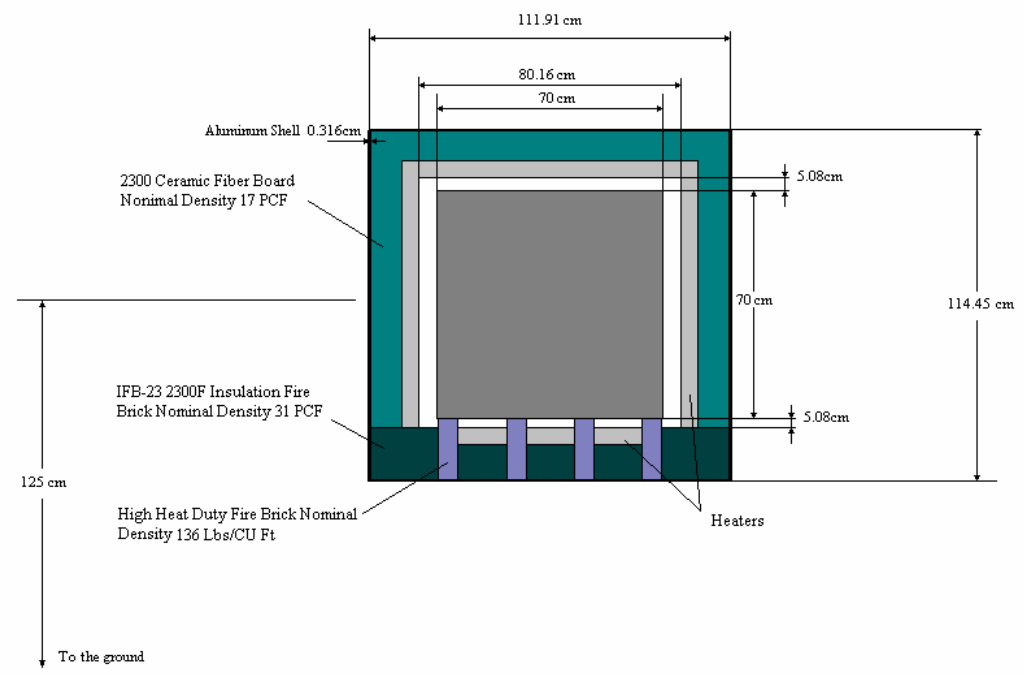

Fig. 35. A schematic of the graphite heating system showing materials and dimensions

Figure 36 presents the simulated response of the detector for different experimental conditions. We can compare the simulation of the clean experiment where the pile is not surrounded by the heating system and without room return with the simulations which consider successively the heating system, and room return.

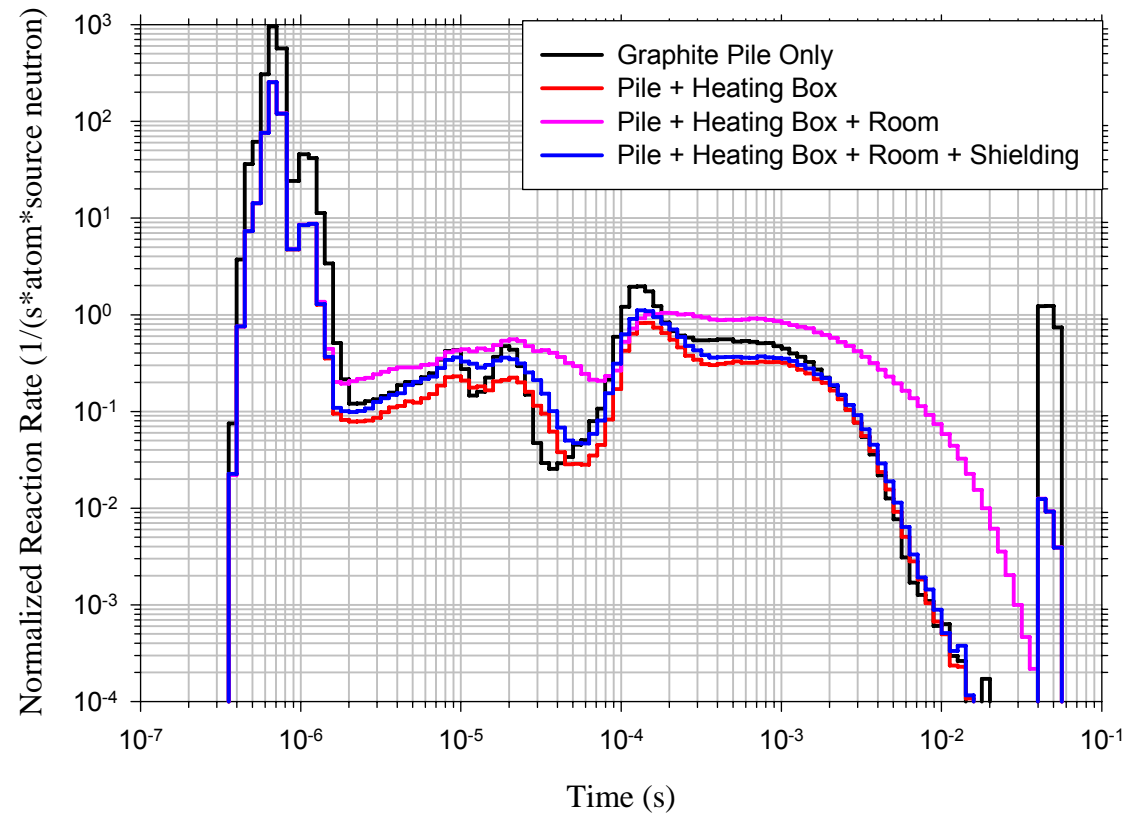

Fig. 36. Simulations at several stages of the design. 
Fig. 37 gives a schematic of the experimental setup including the shielding to protect against room return effects. The shield is composed of 1 -inch thick $5 \%$ borated polyethylene.

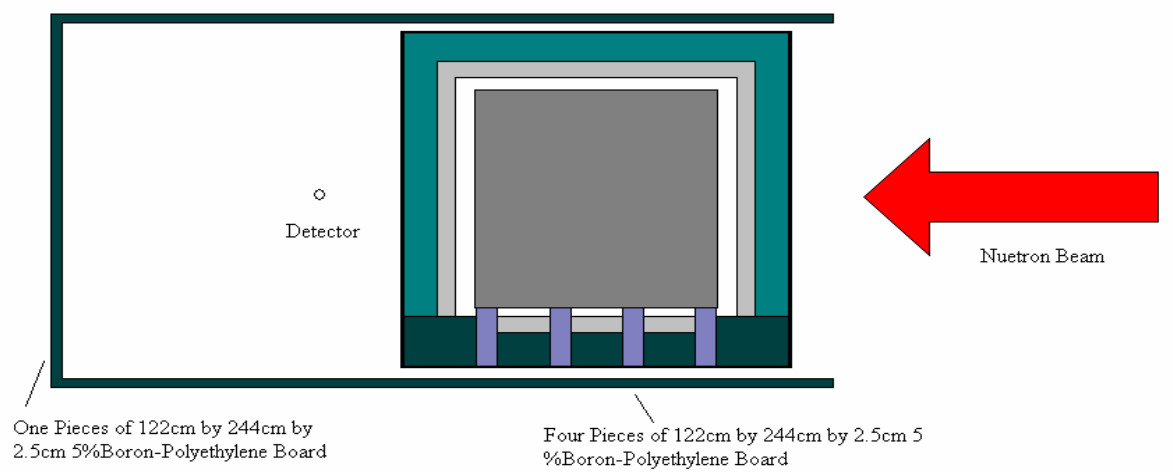

Fig. 37. Optimized shielding of the system.

Figure 38 shows the arrangement of the piles to be measured at room temperature. The designs are based on using the $150 \times 10 \times 10 \mathrm{~cm}^{3}$ rectangular graphite assemblies. To verify how the temperature evolves inside the pile 10 thermocouples will be distributed according to the scheme presented in the figure.

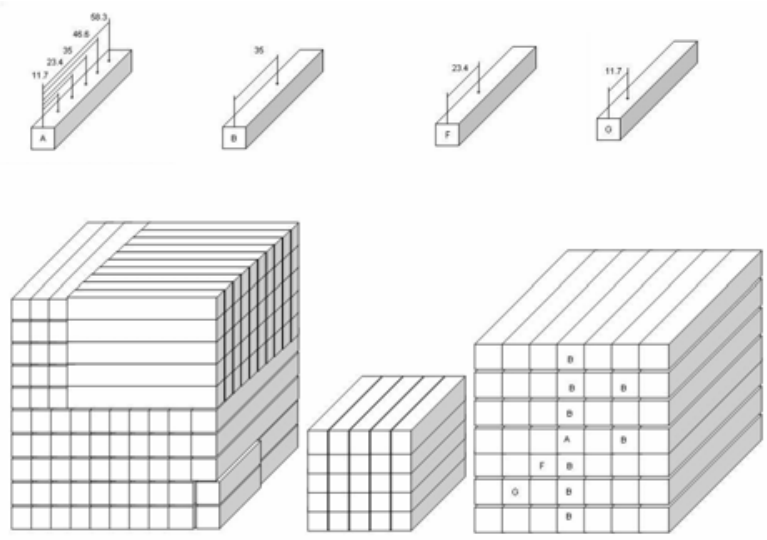

Fig. 38. Arrangement of the graphite piles showing the distribution of the thermocouples.

Based on the above analysis, an experiment report was submitted to ORELA with estimates of the radiation dose surrounding the system. It was found that, during the experiment, all the doses in the positions that 
are off the beam axis remain within acceptable limits. In addition, we discussed the issue of graphite burning and we provided evidence from the literature that this can only happen under extremely rare conditions of temperature and air flow. Therefore, for our enclosed experiment graphite combustion is almost impossible.

Figure 39 below shows an overall diagram of the entire experimental setup as it currently stands at ORELA. In general, all our equipment were moved to ORELA and are set up in the "Electron Room". Computers for data acquisition, and temperature monitoring and control are set up in "Lab A". The communication between the detectors, the temperature control system and the computers is established using Ethernet and BNC connections that are extended between the "Electron Room" and "Lab A".

The heating system table was set up to ensure that the neutron beam is centered on the surface of the graphite pile and is perpendicular to it. The distance from the center of the graphite pile to the wall was measured to be 2 meters. To perform the measurement, two detectors are used. The first is set up on top of the pile and second on the back, 25 $\mathrm{cm}$ from the center of the surface. A $5 \%$ borated polyethylene structure, with $2.5 \mathrm{~cm}$ thick walls and a density of $0.95 \mathrm{~g} / \mathrm{cm}^{3}$, was built to eliminate the background due to neutrons reflecting from the walls and floor of the room. To hold the polyethylene slab and the detectors, a frame, which is made of 14-gauge medium duty slotted angle with dimensions of $2.6 \mathrm{"} \mathrm{x}$ $1.75^{\prime \prime} \times 0.102 " \times 12$ ', was assembled around the furnace table and graphite pile. The polyethylene slabs were cut into 48 " $x 48$ " x 1" pieces and mounted onto the frame. The frame includes two parts: the bell, which holds the polyethylene slab to shield the top detector; and the base which encloses the graphite pile and the back detector. Figure 40 shows the structure of the frame, and Figure 41 shows the picture of the frame and the furnace table. Figure 42 shows the picture of the assembled system in position at the end of the ORELA beam line including the borated polyethylene structure surrounding the graphite heating system.

For the high temperature measurements, 10 thermocouples will be distributed inside the graphite pile to monitor the temperature, as shown in Fig. 43. An additional thermocouple will be placed on top of the furnace to monitor the temperature outside the furnace, and another will be placed under the furnace table to monitor the temperature of the polyethylene slab, which has a melting point of approximately $90^{\circ} \mathrm{C}$. The 12 thermocouples connect to a Yokogawa MV100 temperature monitor. The MV100 can record the temperatures of 12 channels and work as a web server with an Ethernet port equipped to instantly send the temperature data to Lab A. In addition, the MV100 can also be controlled 
by the computer in Lab $A$ by using an interface based on the LabView software. Figure 44 shows testing of the thermocouple system.

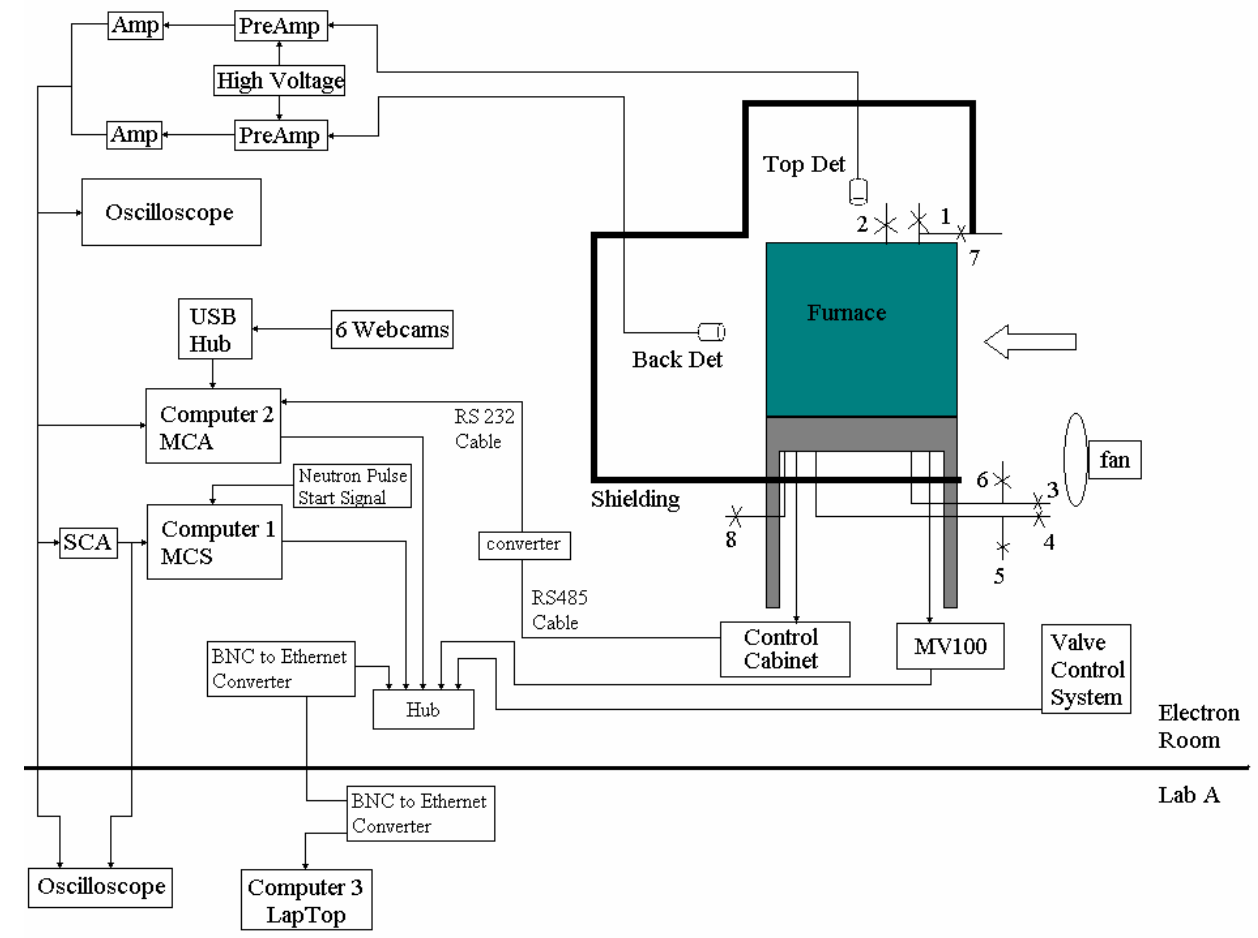

Fig. 39. A schematic of the graphite experiment as it currently stands at ORELA.

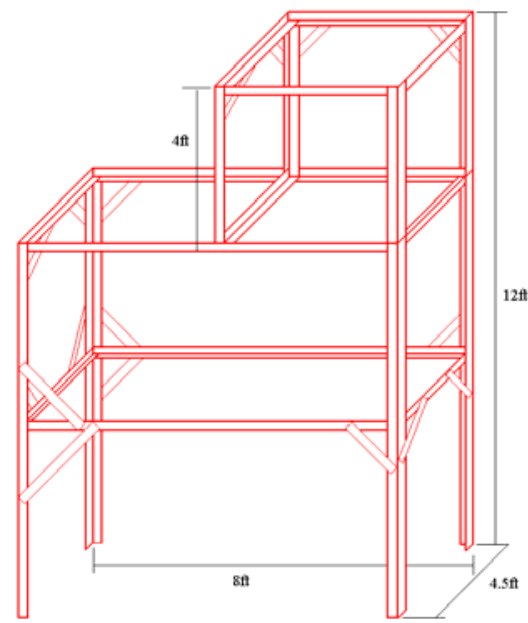

Fig. 40. A schematic of the frame that holds the polyethylene. 


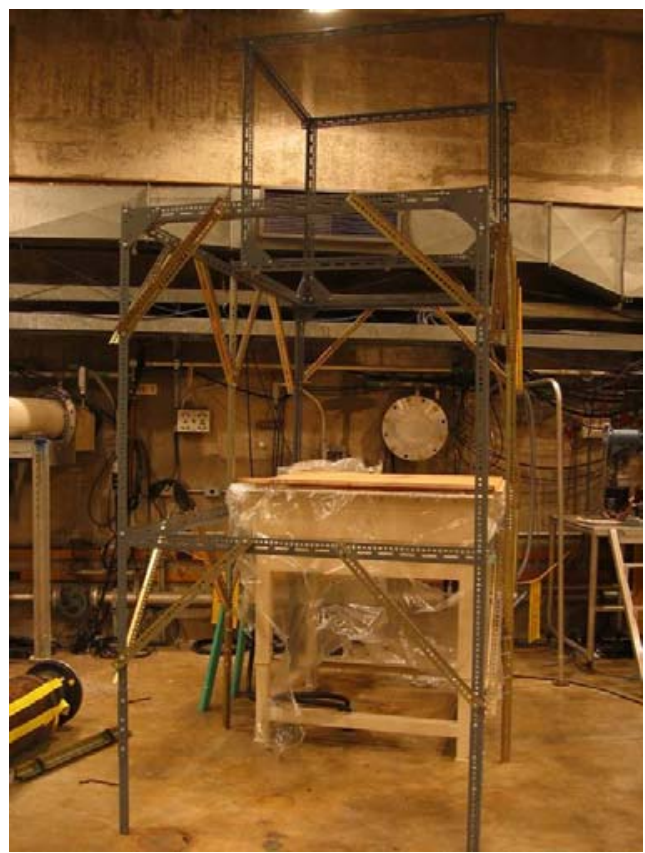

Fig. 41. The graphite heating system table and the frame in position in the electron room.

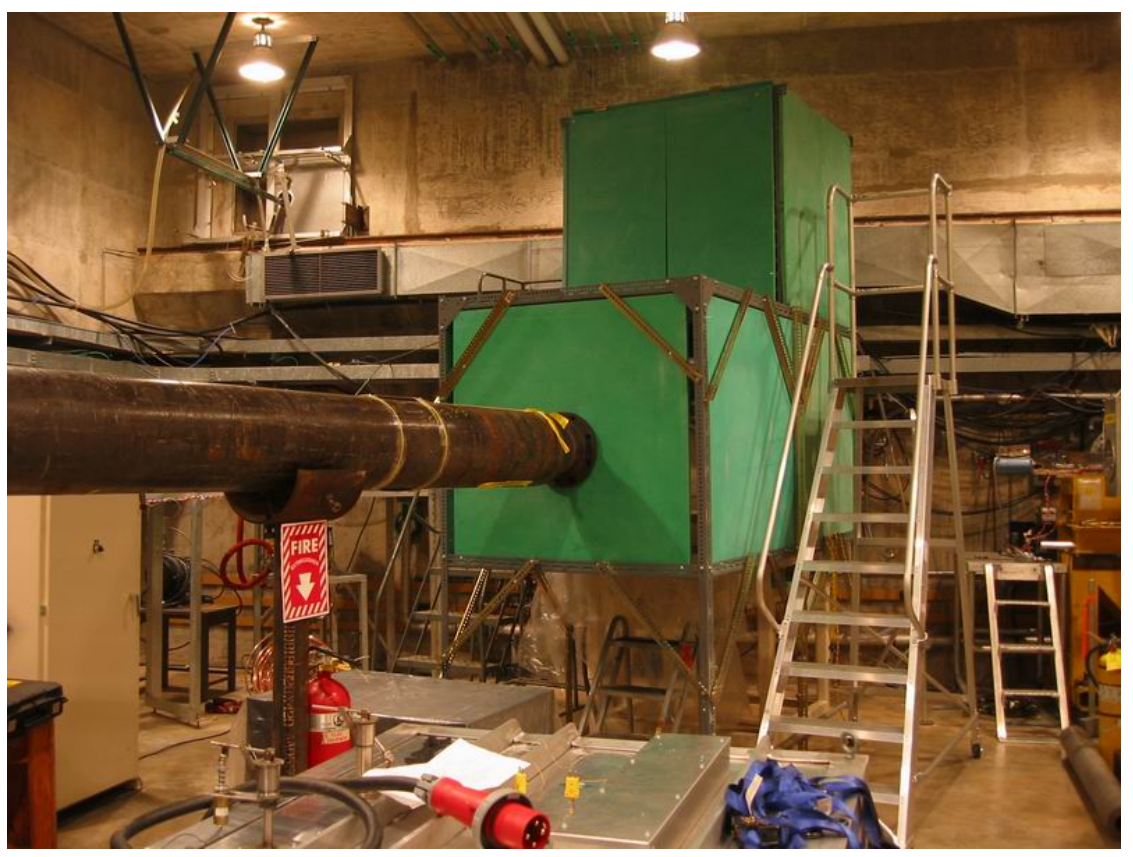

Fig. 42. The assembled experimental system showing the polyethylene shielding structure (green) surrounding the graphite heating system. 


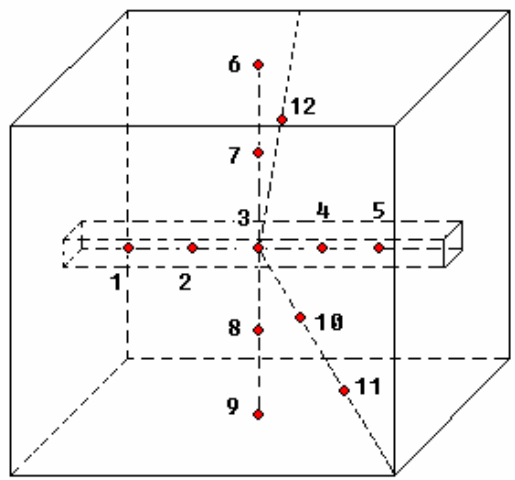

Fig. 43. Thermocouples distribution in the graphite pile. The pile is $70 \times 70 \times 70 \mathrm{~cm}$.

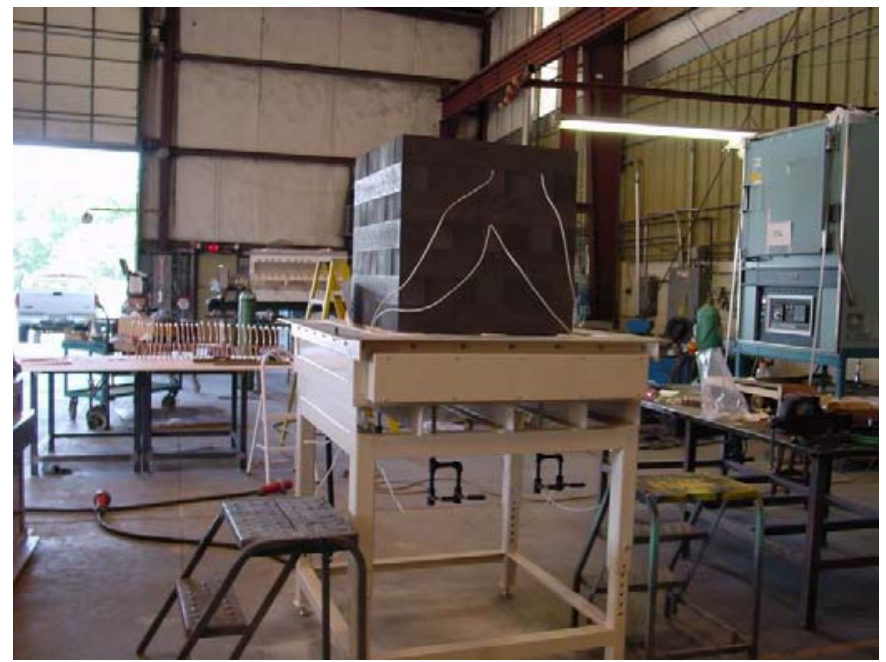

Fig. 44. Testing of the thermocouple system.

As described above (Fig. 39), the control and monitoring of the experiment is performed using a computer based system. An MCA computer and MCS computer were set up in the Electron Room. Using an Ethernet hub in the Electron Room, all the computers including the laptop in Lab A and the equipment (MV100 and FieldPoint PLC for the Valve system) can communicate with each other. In addition, six webcams are connected to the two computers through a USB hub, which can give a close view of the Electron Room.

The two Timing SCA, Amplifier, High Voltage supplies and NIM bins were setup in Electron Room. The Li-6 detectors were connected and mounted in the detector holder. The high voltage for the 5-inch BC702 
detector is $800 \mathrm{~V}$, for the 3 -inch GS20 detector is $1000 \mathrm{~V}$. A background test experiment was performed by using the 3-inch Li-6 detector while ORELA is down. A neutron spectrum was recorded using the MCA and MCS. The background for the detector inside the shielding house was found to be $29 / \mathrm{min}$, and for the detector outside the shielding house it is $34 / \mathrm{min}$. The conclusion is that the measured background is mainly due to gamma-rays.

\section{Measurements and Analysis of Results}

The bare $70 \times 70 \times 70 \mathrm{~cm}$ graphite assembly sitting on the furnace table was used for the room temperature measurements. The measurement setup is as shown in Fig. 39 without including the furnace. As previously described, the configuration for the $5 \%$-boron polyethylene shielding was optimized by MCNP simulations to minimize the background due to neutron room return. The same Li-6 glass scintillation detector was centrally located $45 \mathrm{~cm}$ from the top of the graphite assembly for "topdetector measurements" and $45 \mathrm{~cm}$ from the back of the graphite assembly for "back-detector measurements." In preliminary ORELA runs, the $\mathrm{Li}-6 \mathrm{ZnS}(\mathrm{Ag})$ scintillation detector showed poor timing characteristics and was not used. The U-235 proportional counter also was not used for final measurements due to its low counting rate relative to the ORELA running time available to us. However, preliminary runs with this detector verify timing calibration; the time locations of the structure due to U-235 resonances present in the measured and simulation slowing-down-time distributions agreed within experimental error. Detector pulse-height spectra were collected in a computer based multichannel analyzer (MCA). Simultaneously, slowing-down-time distributions were collected in a multichannel scaler (MCS) in a second computer. The start signal for the MCS was taken from ORELA, and the stop signal was provide by a timing single-channel analyzer fed by the output of the scintillation detector amplifier (MCA input). The settings for the various electronic components of the data acquisition system were determined from parametric measurements during preliminary runs and checked during the final measurements.

The MCNP5 code was utilized to simulate the slowing-down-time experiment. A very detailed MCNP model, previously described, consisted of the $70 \times 70 \times 70 \mathrm{~cm}$ bare graphite assembly, the furnace table, the $5 \%$-boron polyethylene shielding structure, the Li- 6 glass scintillation detector, and two steel detector holders. Also included in the model were a large iron pipe located behind the shielding structure, which was required for radiation safety, and the concrete room in which the measurements were performed (Electron Room). The ENDF/B-VI crosssection data were used for the simulations. The neutron source energy 
distribution used for the simulations was one given to us by the ORELA staff [35]. ORELA was pulsed at a repetition rate of $130 \mathrm{pps}$. This rate was slower than the normal rate used for ORELA experiments, but was required to eliminate pulse overlap, i.e., source pulses occurring before the die away of the neutron detection events from the previous pulse. A rate of $130 \mathrm{pps}$ gives a time interval between pulses of $7.69 \mathrm{~ms}$. The MCS was set to have a channel dwell time of $0.9 \mu \mathrm{s}$ and a full scale of 8192 channels. This gave a MCS full-scale time interval of $7.37 \mathrm{~ms}$, slightly smaller than the time interval between pulses.

The simulated counts for the Li-6 glass scintillation detector were determined by multiplying track-length-estimator tallies of neutron flux, sorted by energy and time, by the appropriate Li- 6 cross sections. The results, obtained by summing over energy, were then sorted into time bins having equal log-time intervals with 20 bins per decade. The measured scintillation detector counts per time channel were grouped into time bins having the same bin structure. When one time channel contributed to two bins, at bin boundaries, the counts in that channel were prorated according to the fraction of channel time in each bin. When two or more time bins fell into one channel, at very short times, the channel count was divided between the bins according to the time width of each bin. The zero of the slowing-down-time scale for the simulations was defined as the time that neutrons, in a Dirac delta function, left the ORELA target. Thus, this time scale included the flight times of neutrons in the flight path. For the measurements, the zero of the time scale was taken to be the center of the one channel, 900 ns wide, that contained a large number of counts from the gamma flash. This was based on the width of the ORELA source pulse being $<30$ ns and the flight time of the gammas down the flight tube being $\sim 30 \mathrm{~ns}$. For purposes of comparing simulated counts to measured counts, the simulation was normalized to have the same number of counts as the measured distribution from $9.46 \mathrm{x}$ $10^{-3} \mathrm{~ms}$ to $4.22 \times 10^{-2} \mathrm{~ms}$. This time interval corresponds to an energy interval of approximately $10 \mathrm{eV}$ to $400 \mathrm{eV}$. In this energy interval and above, the graphite cross sections are well known, and thus the MCNP simulations should give accurate results

The results of a one-hour top-detector measurement showed good agreement with a top- detector simulation for slowing-down time less than $0.3 \mathrm{~ms}(>0.1 \mathrm{eV})$. For times greater than $0.3 \mathrm{~ms}$, the measured counts increase above the simulated counts becoming unreasonably large for times greater than $2 \mathrm{~ms}$. For quantitative differences, see Table 1, 2nd column, "Percent difference, no front shield, no beam filter." The results of a one-hour back-detector measurement showed good agreement with a back-detector simulation for slowing down times less than $0.06 \mathrm{~ms}$ (> 6 $\mathrm{eV}$ ). For times between $0.06 \mathrm{~ms}$ and $1.5 \mathrm{~ms}$, the measured counts lie 
below the simulated counts. For times greater than $1.5 \mathrm{~ms}$, the measured counts increase above the simulated counts becoming unreasonably large for times greater than $4 \mathrm{~ms}$. For quantitative differences, see Table 1, 2nd column, "Percent difference, no front shield, no beam filter." The measured counts being unreasonably larger than expected for long times led us to suspect that thermal neutrons were leaking out of the front of the shielding structure, into the room, scattering around in the equipment in the room, and then back into the front of the shielding structure. The front wall of the lower portion of the shielding structure had been left off because simulations showed that a significant fraction of the thermal neutrons detected by the top detector were scattered off of this front wall. However, the simulations did not include neutron scattering off of equipment near the front of the shielding structure.

Table 1. Comparison between measured counts and simulated counts (Measured Counts - Simulated Counts) / Simulated Counts

Top Detector

\begin{tabular}{|c|c|c|c|}
\hline $\begin{array}{c}\text { Slowing } \\
\text { Down Time } \\
\text { (ms) }\end{array}$ & $\begin{array}{c}\text { Percent difference } \\
\text { no front shield } \\
\text { no beam filter }\end{array}$ & $\begin{array}{c}\text { Percent difference } \\
\text { front shield } \\
\text { no beam filter }\end{array}$ & $\begin{array}{c}\text { Percent difference } \\
\text { front shield } \\
\text { B-10 beam filter }\end{array}$ \\
\hline 0.16 & $\sim 0$ & $\sim 0$ & $\sim 0$ \\
\hline 0.4 & 5 & 5 & $\sim 0$ \\
\hline 1.0 & 20 & 32 & 18 \\
\hline 2.5 & 50 & 70 & 25 \\
\hline 6.25 & 330 & 500 & $\sim 0$ \\
\hline
\end{tabular}

Back Detector

\begin{tabular}{|c|c|c|c|}
\hline $\begin{array}{c}\text { Slowing } \\
\text { Down Time } \\
(\mathrm{ms})\end{array}$ & $\begin{array}{c}\text { Percent difference } \\
\text { no front shield } \\
\text { no beam filter }\end{array}$ & $\begin{array}{c}\text { Percent difference } \\
\text { front shield } \\
\text { no beam filter }\end{array}$ & $\begin{array}{c}\text { Percent difference } \\
\text { front shield } \\
\text { B-10 beam filter }\end{array}$ \\
\hline 0.16 & -25 & -25 & -25 \\
\hline 0.4 & -23 & -25 & -27 \\
\hline 1.0 & -9 & -12 & $\sim 0$ \\
\hline 2.5 & 17 & 17 & 20 \\
\hline 6.25 & 300 & 300 & $\sim 0$ \\
\hline
\end{tabular}




\section{B. Final Measurements with Front Shielding Wall}

A front wall was added to the lower portion of the shielding structure, having the same thickness and composition as the other shielding pieces. The one-hour measurements were repeated and new simulations were performed for this different shielding configuration. The results are given in the 3rd column of Table 1 labeled "Percent difference, front shield, no beam filter." The change in the shielding structure did not decrease the differences between the measured counts and simulated counts. It increased them in a systematic way for the top detector and had little effect on the ratios for the back detector. Thus our explanation for the differences between measured counts and simulated counts was not correct. The main effect of adding a front shielding wall was not to remove non-simulated neutrons scattered back into the shielding structure, but to enhance the reason for the differences between measured counts and simulated counts. The total results to date led us to hypothesize that the neutron source energy distribution given to us by ORELA staff was not correct for the flight path used for our experiment. Simulations with the source distribution arbitrarily changed by increasing the thermal peak by a factor of 20 and the epithermal neutrons in a consistent way agreed moderately well with the measurements. Thermal neutrons are produced in the water that cools the ORELA target. The relative amount of these neutrons in the source depends on the size and position of a shadow bar that may be present to diminish the gamma flash coming directly from the ORELA target.

\section{Final Measurements with a B-10 Beam Filter and Shielding Wall}

A filter containing $0.0934 \mathrm{~g} / \mathrm{cm}^{2}$ of B-10 was placed in the ORELA beam to eliminate the thermal-neutron component of the neutron source. The ability to place certain fixed filters in neutron flight paths is a standard feature of ORELA beams. The one-hour measurements were repeated with the B-10 filter in place. The results were compared to the simulation performed for section $B$ above. The results are given in the 4th column of Table I labeled "Percent difference, front shield, B-10 beam filter." Removing thermal neutrons from the ORELA source greatly reduced the differences between measured counts and simulated counts at long times. This is reasonable since source thermal neutrons have thermalneutron flight times added onto diffusing times.

Figure 45 shows the measured and simulated results along with measured counts without the B-10 filter and measured counts with the Li6 glass scintillation detector covered with $\mathrm{Cd}$. The effect of placing the B10 filter in the neutron flight path is clearly shown in detail in the figure. 
The large decrease in counts at long times is consistent with the absorption of source thermal neutrons, which have flight times of $\sim 3.2 \mathrm{~ms}$ and diffusing times of $\sim 1.5 \mathrm{~ms}$. However, the thermal neutron component in the ORELA B-10 filtered neutron beam was much smaller than the small thermal neutron peak in the neutron source used for the simulations. Removing the small thermal neutron peak from the neutron source used for the simulations would lower the simulated counts at long times. The measured counts at $\sim 6$ ms would then be about $15 \%$ to $25 \%$ above the simulations for both the top and back detector measurements, similar to the differences at $2.5 \mathrm{~ms}$. The measured results shown in Fig. 45 for the detector covered with $\mathrm{Cd}$ show $\mathrm{Cd}$ cutoffs $(0.6 \mathrm{eV})$ at slowing down times consistent with the timing calibrations used for the top detector and the back detector.

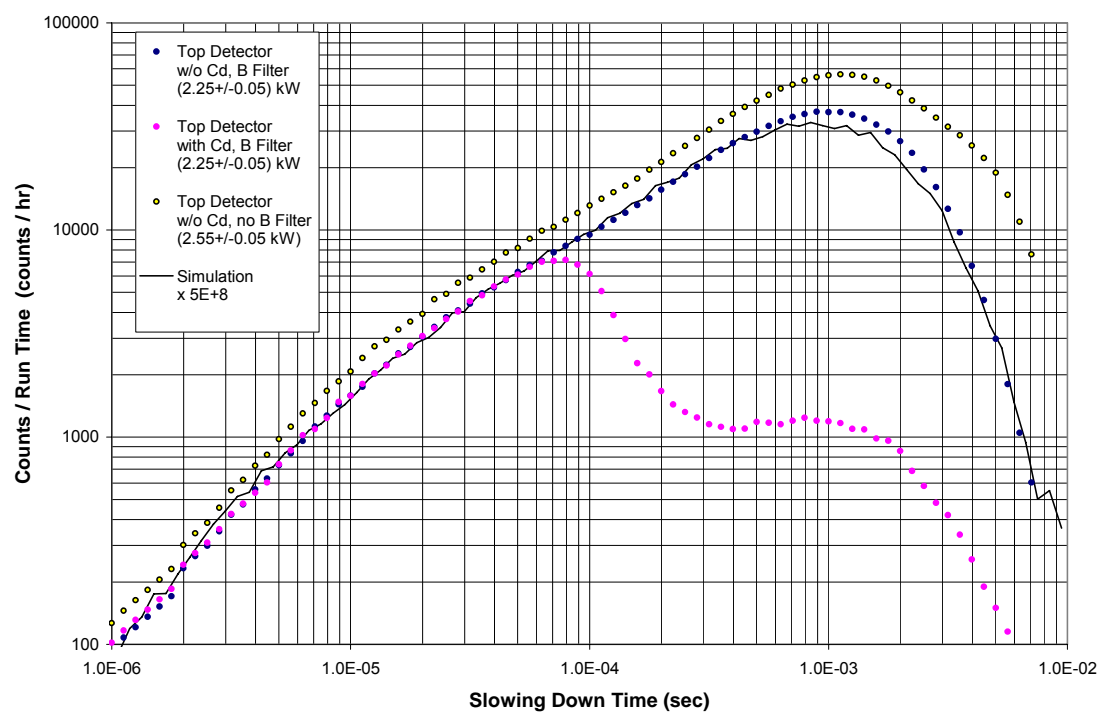

Fig. 45. Measured slowing-down-time distributions for the cases with and without the B-10 filter in the neutron flight path, with and without Cd covering the Li-6 glass scintillation detector, and compared with the simulation done with a small thermal-neutron peak $(\sim 20$ times too small) and no Cd covering the detector. The simulation used ENDF/BVI cross-section data. The "with Cd" data set clearly illustrates the time-energy coupling effect in this experiment and shows that at times around 1.0E-4 seconds the neutrons have reached an energy near the Cd cut-off $(\sim 0.5 \mathrm{eV})$. At longer times, the neutrons will have energies below $0.5 \mathrm{eV}$. 


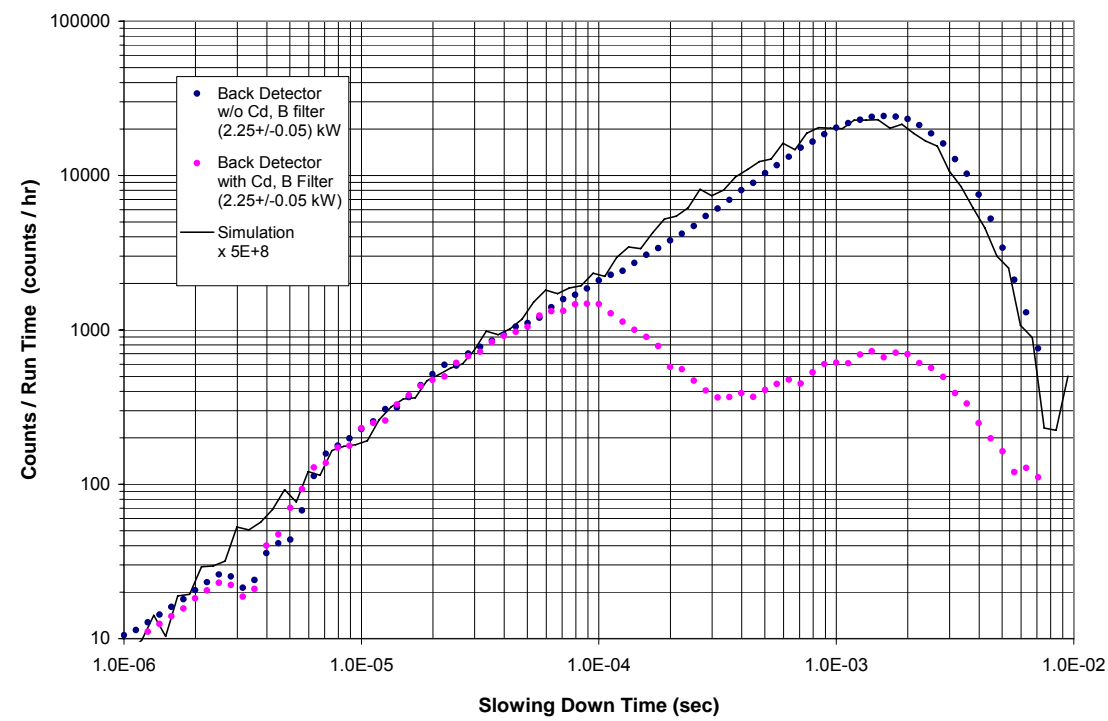

Fig. 45. Continued

For the top detector, the measured counts agree reasonably well with the simulated counts for slowing down times less than $0.5 \mathrm{~ms}$, but are roughly $20 \%$ higher than the simulated counts for slowing down times greater than $1 \mathrm{~ms}$. For the bottom detector, the measured counts agree reasonably well with the simulated counts for slowing down times less than $0.05 \mathrm{~ms}$, are roughly $25 \%$ bellow the simulated counts for slowing down times between $0.15 \mathrm{~ms}$ and $0.5 \mathrm{~ms}$, and are roughly $20 \%$ above the simulated counts for slowing down times grater than $3 \mathrm{~ms}$. A great deal of effort was spent on investigating systematic errors in the measurements. Errors in time calibration, detector positions, scintillator thickness, and source pulse overlap were eliminated as causing the differences given above. The effect of the gamma flash on the Li- 6 glass scintillation detector was also ruled out.

To gain insight into the reasons for the differences given above, simulations were carried out having counts also sorted according to the number of neutron collisions in the graphite. The results are shown in Fig. 46 for selected number-of-collision intervals. Neutrons in number-ofcollision intervals 1 - 10, 10 - 20, and 20 - 40 were counted after escaping from the assembly while slowing down in the graphite; for these neutron counts, there is a direct correlation between neutron detection time and neutron energy. Neutrons in number-of-collision intervals $100-200,200$ - 400, and 400 - 800 were counted after escaping from the assembly while diffusing in the graphite; for these neutron counts, there is little correlation between neutron detection time and neutron energy. The shapes and time locations of the time distributions with the same number- 
of-collisions were remarkably similar for the top and back detectors. but the number of counts under these time distributions were different.

Figure 46 shows that, relative to the number of counts of neutrons escaping the assembly while diffusing, significantly more neutrons were counted by the top detector after escaping the assembly while slowing down than were counted by the back detector. It is to be expected that more neutrons would escape the top of the assembly, while slowing down, than would escape the back of the assembly, while slowing down, due to the longer graphite path to the back detector. It follows then that, in the time interval $0.15 \mathrm{~ms}-0.5 \mathrm{~ms}$, the top detector counted mostly neutrons that escaped while slowing down while the back detector counted mostly neutrons that escaped while diffusing. Thus, we conclude that the differences between the measured and simulated counts for the back detector in the time interval $0.15 \mathrm{~ms}-0.5 \mathrm{~ms}$ are due to errors in the ENDF/B-VI thermal neutron scattering cross sections. The better agreement between the measured and simulated counts for the top detector in the same time interval reflects better accuracy for the neutron scattering cross sections in the slowing down energy region. Errors in the thermal neutron scattering cross sections also account for the differences between the measured and simulated counts for both detectors for neutron detection times greater than $1 \mathrm{~ms}$.

The above arguments are based on simulations. However, the shapes of the measured pulse-height spectra, shown in Fig. 47 are consistent with these arguments. The pulse-height peaks measured by both the top and back detectors appear to be the sum of two components. One component is a Gaussian at about channel 500 due to the detection of thermal neutrons $(Q$ value $4.78 \mathrm{MeV})$. The other component is a smeared out Gaussian due to the detection of neutrons with energies below $1 \mathrm{MeV}$, i.e., slowing down neutrons, peaked at about channel 526 due to a Li- 6 neutron resonance at $250 \mathrm{keV}$. The response of the top detector covered with $\mathrm{Cd}$ shows a small thermal-neutron component (thermal neutrons leaking into the detector) and a larger slowing down component. The response of the back detector covered with $\mathrm{Cd}$ shows a small thermal- neutron component, similar in size to the thermal-neutron component of the top detector, but no slowing-down component. 

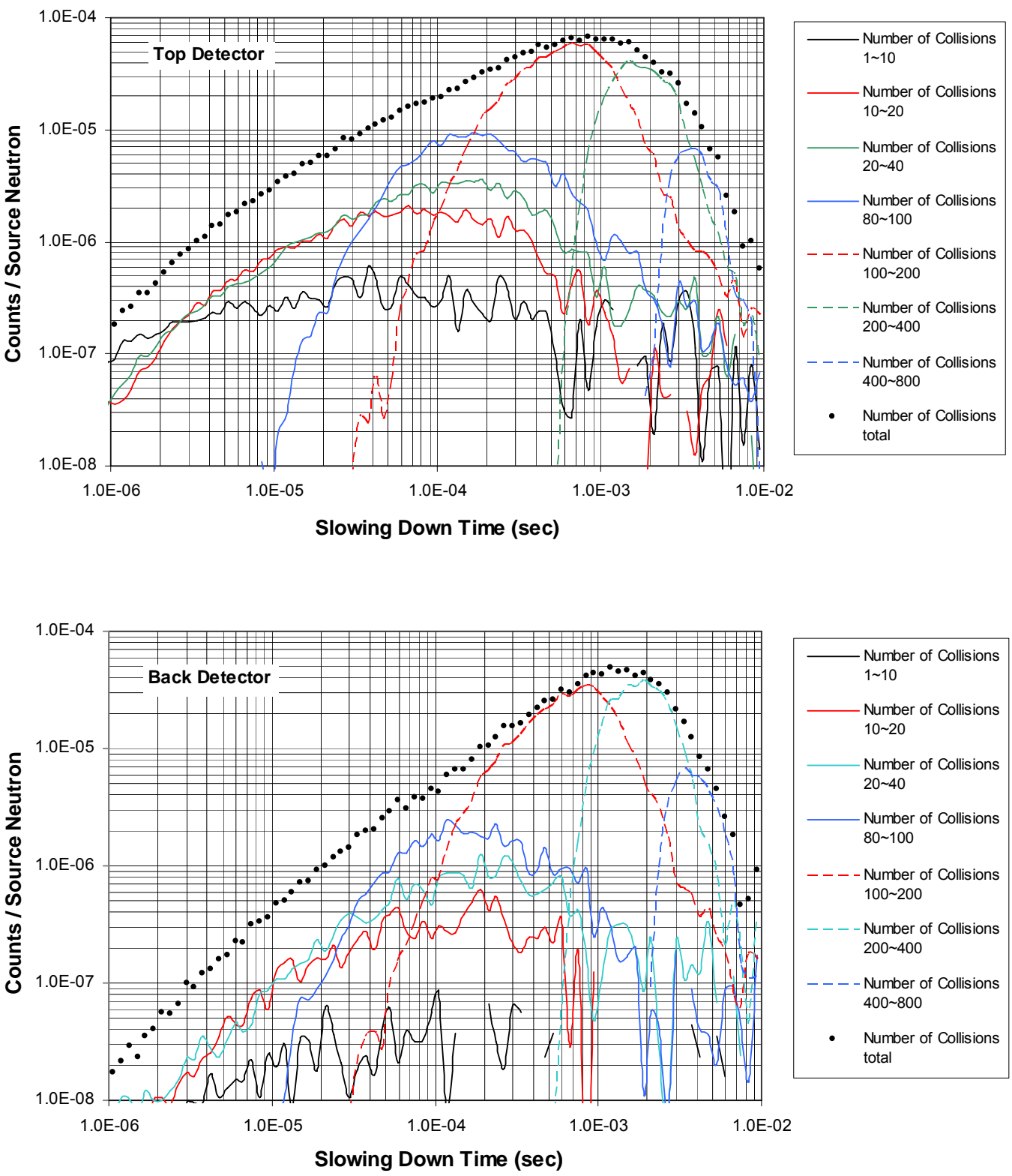

Fig. 46. MCNP simulated time distributions sorted according to number of neutron collisions in the graphite. The neutrons in number-of-collision intervals $1-10,10-20$, and $20-40$ were counted after escaping from the assembly while slowing down in the graphite; the neutrons in number-ofcollision intervals $100-200,200-400$, and $400-800$ were counted after escaping from the assembly while diffusing in the graphite. 

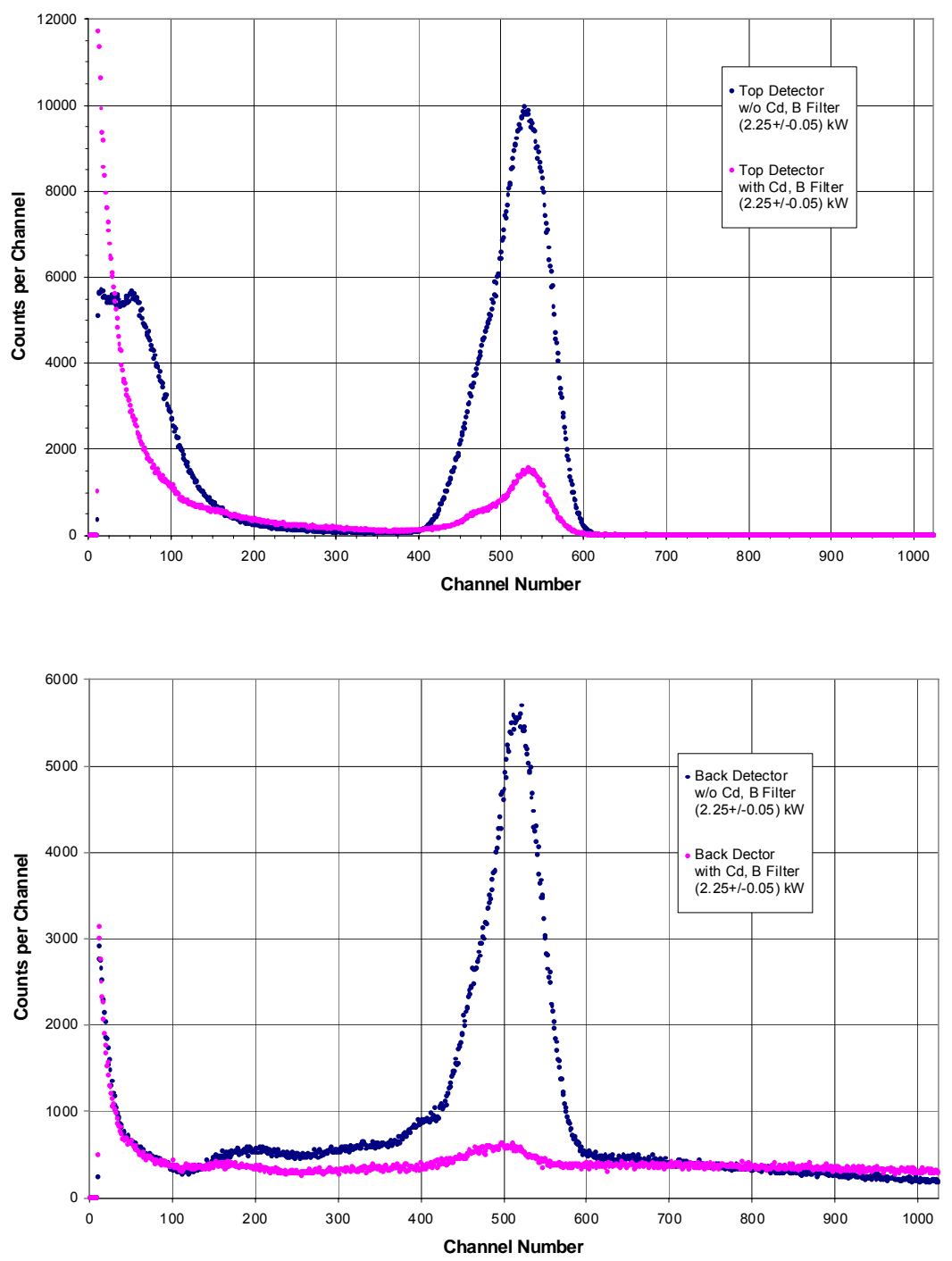

Fig. 47. Pulse-height spectra for the Li-6 glass scintillation detector measured with the B-10 filter in the neutron flight path.

\section{References}

1) Squires, G. L., Introduction to the Theory of Thermal Neutron Scattering, Cambridge University Press, UK, 1977.

2) Koppel, U., Triplett, J. R. and Naliboff, Y. D., GASKET: A Unified Code for Thermal Neutron Scattering, General atomic report, GA-7417, 1967. 
3) MacFarlane, R. E., and Muir, D. W., The NJOY Nuclear Data Processing System, Version 91, LA-12740-MS, Los Alamos National Laboratory, 1994.

4) Chadwick, M. B., et al., Nuclear Data Sheets, v. 107, 2931, 2006.

5) Steyrel, A., and Trustedt, W. D., Z. Physik, v. 267, 379, 1974.

6) Borgonovi, G. M., and Sprevak, D., Nuclear Science and Engineering, v. 42, no. 2, 137, 1970.

7) Maradudin, A. A., Montroll, E. W., Weiss, G. H., and Ipatova, I. P., Theory of Lattice Dynamics in the Harmonic Approximation, Academic Press, New York, 1971.

8) Hohenberg, P., and Kohn, W., Phys. Rev., v. 136, B864, 1964.

9) Kohn, W., and Sham, L. J., Phys. Rev., v. 140, A1133, 1965.

10) Feynman, R. P., Physical Review, v. 56, 340, 1939.

11) Kresse, G., and Furthmuller, J., "Vienna Ab-Initio Simulation Package, VASP the Guide," Vienna, 2002.

12) Parlinski K., "PHONON manual, version 3.11," Krakow, 2002.

13) Lazzeri, M., and De Gironcoli, S., Surf. Sci., 402-404, 715, 1998.

14) Young, J. A., and Koppel, J. U., Phys. Rev., 6, A1476, 1964.

15) Hughs, D. J., and Shwartz, R. B., Neutron Cross Sections-BNL 325, Second Edition, 1958.

16) Benedek, G., Hofmann, F., Ruggerone, Onida, G., and Miglio, L., Solid State Comm., v. 65, 1601, 1988.

17) Maultzsch, J., Reish, S., Thomson, C., Requardt, H., and Ordejon, P., Phys. Rev. Lett., v. 92, 075501-1, 2004.

18) Nicklow, R., and Wakabayashi, N., and Smith, H. G., Phys. Rev. B, v. 5, 4951, 1972.

19) Oshima. C., Aizawa, T., Souda, R., Ishizawa., $Y$, and Sumiyoshi, Y., Solid State Commun, v. 65, 1601, 1988.

20) Siebentritt, S., Pues, R., and Rieder, K., Phys. Rev. B, v. 55, 7927, 1997.

21) Wilkes, J. L., Palmer, R. E., and Willis, R. F., J. Electron Spectrosc. Relat. Phenom., v. 44, 355, 1987.

22) Young, J. A., and Koppel, J. U., J. Chem. Phys., v. 42, 357, 1965.

23) Zhou, T., Benchmarking Thermal Neutron Scattering in Graphite, Ph.D. Dissertation, North Carolina State University, 2007.

24) Edura, $Y$. , and Morishima, N., Nuclear Instruments and Methods in Physics research-A, v. 534, Issue 3, 531-543, 2004.

25) Borgonovi, G., Lattice Dynamics and Neutron Scattering of $\mathrm{BeO}$, General Atomic report GA-8758, 1968.

26) Lin and Keoing, J. Molec. Spectra, 9, 228, 1962. 
27) Sprevak and Koppel, Nucleonik, 12, 87, 1969.

28) Swaminathan and Tewari, Nucl. Sci. Eng., 91, 95, 1985.

29) Lake, J. A., and Kallfelz, J. M., Nuclear Science and Engineering, 53, 27-46, 1974.

30) Watanabe, N. et al., J. Nuclear Energy, 25, 203, 1971.

31) Young, J.C., G. D. Trimble, Y. D. Naliboff, G. H. Houston and J. R. Beyster, Nucl. Sci. Eng., 18, 376-399, 1964.

32) McReynolds, A. W., M. S. Nelkin, M. N. Rosenbluth, and W. L. Whitmore, "Neutron Thermalization by Chemically-bound Hydrogen and Carbon," Proc. 2nd U. N. Conf. Peaceful Uses At. Energy, 16, 297, 1958.

33) Young, J. C., J. A. Young, G. K. Houghton, G. D. Trimble, and J. R. Beyster, Nucl. Sci. Eng., 19, 230, 1964.

35) ORELA Staff, private communications, 2003. 


\section{Related Publications}

Several publications related to this project were produced:

2. I. I. Al-Qasir, Ayman I. Hawari, "Graphite Thermal Neutron Cross Section Calculations Including Coherent 1-Phonon Effects," Transactions of the American Nuclear Society, 96, 2007.

3. T. Zhou, Ayman I. Hawari, B. W. Wehring, "Utilizing the SlowingDown-Time Technique for Benchmarking Neutron Thermalization in Graphite," Proceeding of the 13th international Conference on Emerging Nuclear Energy Systems, Istanbul, Turkey, 2007.

4. Y. Cao, T. Zhou, I. I. Al-Qasir, Ayman I. Hawari, R. F. Fleming, J. C. Lee, "MCNP5 Simulations of ZrH Scattering Experiments," Transactions of the American Nuclear Society, 94, 2006.

5. T. Zhou, Ayman I. Hawari, B. W. Wehring, V. H. Gillette, I. I. Al-Qasir, "Benchmark for Beryllium Thermal Neutron Scattering Cross Sections," Transactions of the American Nuclear Society, 91, 2004.

6. Ayman I. Hawari, I. I. Al-Qasir, V. H. Gillette, B. W. Wehring, T. Zhou, "Ab Initio Generation of Thermal Neutron Scattering Cross Sections," Proceedings of the PHYSOR conference, Chicago, IL, 2004. (Invited)

7. T. Zhou, Ayman. I. Hawari, B. W. Wehring, I. I. Alqasir, V. H. Gillette, F. C. Difilippo, J. P. Renier, "Utilizing The Oak Ridge Electron Linear Accelerator Facility For Benchmarking Neutron Thermalization in Moderators," Proceedings of AccApp'03 conference, San Diego, California, 2003.

8. I. I. Al-Qasir, Ayman I. Hawari, B. W. Wehring, V. H. Gillette, Tong Zhou, F. C. Difilippo, J.P. Renier, "Generation of Thermal Neutron Scattering Cross Sections Using $\mathrm{Ab}$ Initio Phonon Frequency Distributions," Transactions of the American Nuclear Society, 88, 2003.

9. Ayman I. Hawari, Tong Zhou, Bernard W. Wehring, Felix C. Difilippo, John-Paul Renier, "Simulated Experiment for Benchmarking the Thermal Neutron Scattering Law in Graphite at the ORELA Facility," Transactions of the American Nuclear Society, 87, 468, 2002. 
10. Ayman I. Hawari, lyad Al-Qasir, Bernard W. Wehring, Felix C. Difilippo, John-Paul Renier, "Investigation of Neutron Thermalization in Graphite Moderators," Transactions of the American Nuclear Society, 87, 470, 2002.

11. F. C. Difilippo, J. P. Renier, and Ayman I. Hawari, "Benchmarks for the Scattering Kernel of Graphite," Proceedings of the PHYSOR conference, Seoul, Korea, 2002. 
Final Project Report

DE-FG07-02SF22514

June 20, 2008

Milestone Status Table:

\section{Phase 1}

\begin{tabular}{|c|c|c|c|c|}
\hline $\begin{array}{c}\text { ID } \\
\text { Number }\end{array}$ & Task / Milestone Description & $\begin{array}{c}\text { Planned } \\
\text { Completion }\end{array}$ & $\begin{array}{c}\text { Actual } \\
\text { Completion }\end{array}$ & Comments \\
\hline (a) & $\begin{array}{l}\text { Evaluate models and data used in current } \\
\text { calculations of scattering law for the } \\
\text { proposed materials }\end{array}$ & $3 / 31 / 2002$ & $3 / 31 / 2002$ & \\
\hline (b) & $\begin{array}{l}\text { Evaluate improved new models and data } \\
\text { relevant to the thermalization problem in } \\
\text { neutron and condensed matter physics }\end{array}$ & $5 / 31 / 2002$ & $5 / 31 / 2002$ & \\
\hline (c) & $\begin{array}{l}\text { Start the implementation of new } \\
\text { computational tools to calculate with new } \\
\text { models and/or data }\end{array}$ & $5 / 31 / 2002$ & $5 / 31 / 2002$ & \\
\hline (d) & $\begin{array}{l}\text { Evaluate experimental data available to } \\
\text { define a set of benchmarks }\end{array}$ & $3 / 31 / 2002$ & $3 / 31 / 2002$ & \\
\hline (e) & $\begin{array}{l}\text { Start the development of models to } \\
\text { compute experimental benchmarks }\end{array}$ & $8 / 31 / 2002$ & $8 / 31 / 2002$ & \\
\hline (f) & $\begin{array}{l}\text { Begin designing the graphite benchmark } \\
\text { experiments that will be performed at } \\
\text { ORELA }\end{array}$ & $5 / 31 / 2002$ & $5 / 31 / 2002$ & \\
\hline (g) & $\begin{array}{l}\text { Begin preparation of thermal neutron } \\
\text { scattering libraries }\end{array}$ & $8 / 31 / 2002$ & $8 / 31 / 2002$ & \\
\hline
\end{tabular}

\section{Phase 2}

\begin{tabular}{|c|c|c|c|c|}
\hline $\begin{array}{c}\text { ID } \\
\text { Number }\end{array}$ & Task / Milestone Description & $\begin{array}{l}\text { Planned } \\
\text { Completion }\end{array}$ & $\begin{array}{c}\text { Actual } \\
\text { Completion }\end{array}$ & Comments \\
\hline (a) & $\begin{array}{l}\text { Perform Calculations of graphite } \\
\text { benchmarks }\end{array}$ & $5 / 30 / 2003$ & $9 / 30 / 2003$ & \\
\hline (b) & $\begin{array}{l}\text { Perform Calculations of } \mathrm{Be} \text { and } \mathrm{BeO} \\
\text { benchmarks }\end{array}$ & $5 / 30 / 2003$ & $7 / 30 / 2003$ & \\
\hline (c) & Perform Calculations of $\mathrm{ZrH}$ benchmarks & $5 / 30 / 2003$ & $5 / 30 / 2003$ & \\
\hline (d) & $\begin{array}{l}\text { Continue work on thermal neutron } \\
\text { scattering laws }\end{array}$ & $9 / 30 / 2003$ & $9 / 30 / 2003$ & \\
\hline (e) & $\begin{array}{l}\text { Begin study of effect of scattering law on } \\
\text { safety performance of a given reactor } \\
\text { design }\end{array}$ & $9 / 30 / 2003$ & $9 / 30 / 2003$ & \\
\hline (f) & $\begin{array}{l}\text { Finalize the computational designs for the } \\
\text { graphite experiments, and begin } \\
\text { preparations and setup for performing it }\end{array}$ & $2 / 28 / 2003$ & $5 / 30 / 2003$ & \\
\hline (g) & $\begin{array}{l}\text { Order equipment needed to perform } \\
\text { graphite experiment }\end{array}$ & $2 / 28 / 2003$ & $7 / 30 / 2003$ & \\
\hline
\end{tabular}


Final Project Report

June 20, 2008

DE-FG07-02SF22514

Phase 3

\begin{tabular}{|c|c|c|c|}
\hline $\begin{array}{c}\text { ID } \\
\text { Number }\end{array}$ & Task / Milestone Description & $\begin{array}{l}\text { Planned } \\
\text { Completion }\end{array}$ & $\begin{array}{c}\text { Actual } \\
\text { Completion }\end{array}$ \\
\hline (a) & Perform Calculations of $\mathrm{H}_{2} \mathrm{O}$ benchmarks & 05/31/2005 & 05/31/2005 \\
\hline (b) & $\begin{array}{l}\text { Perform Calculations of Polyethylene } \\
\text { benchmarks }\end{array}$ & 05/31/2005 & $05 / 31 / 2005$ \\
\hline (c) & $\begin{array}{l}\text { Finalize the work and conclusions on } \\
\text { thermal neutron scattering laws }\end{array}$ & $12 / 31 / 2005$ & $09 / 30 / 2005$ \\
\hline (d) & $\begin{array}{l}\text { Finalize study of effect of scattering law on } \\
\text { safety performance of a given reactor } \\
\text { design }\end{array}$ & $12 / 31 / 2005$ & $12 / 31 / 2005$ \\
\hline (e) & $\begin{array}{l}\text { Perform neutron slowing down in the } \\
\text { graphite experiment and compare model } \\
\text { predictions to experimental data }\end{array}$ & $12 / 31 / 2006$ & $12 / 31 / 2007$ \\
\hline (f) & $\begin{array}{l}\text { Perform time-of-flight experiment and } \\
\text { compare results to model predictions }\end{array}$ & $12 / 31 / 2006$ & $05 / 31 / 2005$ \\
\hline
\end{tabular}


Final Project Report

DE-FG07-02SF22514

June 20, 2008

\section{Budget Data:}

The following budget numbers include both the NC State University and Oak Ridge National Laboratory components

\begin{tabular}{|c|c|c|c|c|c|c|c|c|}
\hline \multirow{2}{*}{\multicolumn{3}{|c|}{$\begin{array}{c}\text { NC State University } \\
\text { Phase / Budget Period }\end{array}$}} & \multicolumn{3}{|c|}{ Approved Spending Plan } & \multicolumn{3}{|c|}{ Actual Spent } \\
\hline & & & \multirow{2}{*}{$\begin{array}{c}\text { DOE } \\
\text { Amount }\end{array}$} & \multirow{2}{*}{$\begin{array}{c}\text { Cost } \\
\text { Share }\end{array}$} & \multirow[t]{2}{*}{ Total } & \multirow{2}{*}{$\begin{array}{c}\text { DOE } \\
\text { Amount }\end{array}$} & \multirow{2}{*}{$\begin{array}{c}\text { Cost } \\
\text { Share }\end{array}$} & \multirow[t]{2}{*}{ Total } \\
\hline & From & To & & & & & & \\
\hline Phase 1 & $08 / 01 / 01$ & $12 / 31 / 01$ & $\$ 28,031$ & --- & $\$ 28,031$ & $\$ 28,031$ & --- & $\$ 28,031$ \\
\hline Phase 2 & $05 / 15 / 02$ & $07 / 31 / 03$ & $\$ 318,138$ & --- & $\$ 318,138$ & $\$ 258,520$ & --- & $\$ 258,520$ \\
\hline Phase 3 & $08 / 01 / 03$ & $12 / 31 / 07$ & $\$ 178,868$ & --- & $\$ 178,868$ & $\$ 238,486$ & --- & $\$ 238,486$ \\
\hline & & & & & & & & \\
\hline & & & & & & & & \\
\hline & & & & & & & & \\
\hline & & Totals & $\$ 525,037$ & --- & $\$ 525,037$ & $\$ 525,037$ & --- & $\$ 525,037$ \\
\hline
\end{tabular}

\begin{tabular}{|c|c|c|c|c|c|c|c|c|}
\hline \multirow{2}{*}{\multicolumn{3}{|c|}{$\begin{array}{c}\text { Oak Ridge National } \\
\text { Laboratory }\end{array}$}} & \multicolumn{3}{|c|}{ Approved Spending Plan } & \multicolumn{3}{|c|}{ Actual Spent } \\
\hline & & & \multirow{2}{*}{$\begin{array}{c}\mathrm{DOE} \\
\text { Amount }\end{array}$} & \multirow{2}{*}{$\begin{array}{c}\text { Cost } \\
\text { Share }\end{array}$} & \multirow[t]{2}{*}{ Total } & \multirow{2}{*}{$\begin{array}{c}\mathrm{DOE} \\
\text { Amount }\end{array}$} & \multirow{2}{*}{$\begin{array}{c}\text { Cost } \\
\text { Share }\end{array}$} & \multirow[t]{2}{*}{ Total } \\
\hline & From & To & & & & & & \\
\hline Phase 1 & $10 / 1 / 01$ & 09/30/02 & $\$ 90,444$ & --- & $\$ 90,444$ & $\$ 90,444$ & --- & $\$ 90,444$ \\
\hline Phase 2 & $10 / 1 / 02$ & $09 / 30 / 03$ & $\$ 90,444$ & --- & $\$ 90,444$ & $\$ 90,444$ & --- & $\$ 90,444$ \\
\hline Phase 3 & $10 / 01 / 03$ & $09 / 30 / 05$ & $\$ 90,444$ & & $\$ 90,444$ & $\$ 90,444$ & & $\$ 90,444$ \\
\hline & & & & & & & & \\
\hline & & & & & & & & \\
\hline & & Totals & $\$ 271,332$ & --- & $\$ 271332$ & $\$ 271332$ & --- & $\$ 271332$ \\
\hline
\end{tabular}

